\title{
Kinematic dynamo action in large magnetic Reynolds number flows driven by shear and convection
}

\author{
By YANNICK PONTY, ANDREW D. GILBERT \\ AND ANDREW M. SOWARD
}

School of Mathematical Sciences, University of Exeter, Exeter EX4 4QE, UK

(Received 10 March 2000 and in revised form 22 November 2000)

A numerical investigation is presented of kinematic dynamo action in a dynamically driven fluid flow. The model isolates basic dynamo processes relevant to field generation in the Solar tachocline. The horizontal plane layer geometry adopted is chosen as the local representation of a differentially rotating spherical fluid shell at co-latitude $\vartheta$; the unit vectors $\widehat{\boldsymbol{x}}, \widehat{\boldsymbol{y}}$ and $\widehat{\boldsymbol{z}}$ point east, north and vertically upwards respectively. Relative to axes moving easterly with the local bulk motion of the fluid the rotation vector $\boldsymbol{\Omega}$ lies in the $(y, z)$-plane inclined at an angle $\vartheta$ to the $z$-axis, while the base of the layer moves with constant velocity in the $x$-direction. An Ekman layer is formed on the lower boundary characterized by a strong localized spiralling shear flow. This basic state is destabilized by a convective instability through uniform heating at the base of the layer, or by a purely hydrodynamic instability of the Ekman layer shear flow. The onset of instability is characterized by a horizontal wave vector inclined at some angle $\epsilon$ to the $x$-axis. Such motion is two-dimensional, dependent only on two spatial coordinates together with time. It is supposed that this two-dimensionality persists into the various fully nonlinear regimes in which we study large magnetic Reynolds number kinematic dynamo action.

When the Ekman layer flow is destabilized hydrodynamically, the fluid flow that results is steady in an appropriately chosen moving frame, and takes the form of a row of cat's eyes. Kinematic magnetic field growth is characterized by modes of two types. One is akin to the Ponomarenko dynamo mechanism and located close to some closed stream surface; the other appears to be associated with stagnation points and heteroclinic separatrices.

When the Ekman layer flow is destabilized thermally, the well-developed convective instability far from onset is characterized by a flow that is intrinsically time-dependent in the sense that it is unsteady in any moving frame. The magnetic field is concentrated in magnetic sheets situated around the convective cells in regions where chaotic particle paths are likely to exist; evidence for fast dynamo action is obtained. The presence of the Ekman layer close to the bottom boundary breaks the up-down symmetry of the layer and localizes the magnetic field near the lower boundary.

\section{Introduction}

In this paper we study kinematic dynamo instabilities in flows that are heated from below and contain a localized layer of strong shear at their base, which in our model takes the form of an Ekman layer. Following instabilities of the equilibrium 
basic state, overturning convection occurs; this is driven either by buoyancy forces or by extracting kinetic energy from the shear flow. Though both energy sources act simultaneously the character of the flow is generally dominated by one or other instability mechanism. Depending on the complexity of the ensuing flow, magnetic field growth occurs through slow or fast dynamo mechanisms.

Our study is broadly motivated by the problem of the Solar dynamo. Until recently the consensus was that the generation of the Solar magnetic field arose through differential rotation and convective motions throughout the convection zone of the Sun (Moffatt 1978; Parker 1979; Krause \& Rädler 1980). There were however some large-scale numerical simulations that failed to bear out this picture (Gilman 1983; Glatzmaier 1984, 1985a,b). Then about 10 years ago the understanding of the angular velocity of the Sun was revolutionized by the results of the inversion of helioseismological data. This shows that the inner, radiative zone of the Sun undergoes approximately solid body rotation, while in the convection zone the angular velocity is a function of the latitude (Brown et al. 1989; Dziembowski, Goode \& Libbrecht 1989; Goode et al. 1991). The difference in rotation rate between the convection and radiative zones is taken up in a thin layer of shear, which has been termed the 'tachocline'. This layer has been modelled as a boundary layer in which transport of angular momentum is predominantly horizontal, vertical motions being suppressed by stable stratification (Spiegel \& Zahn 1992).

This led to the suggestion that the seat of the dynamo is not the convection zone itself but instead inside the tachocline (see Roberts 1992; Weiss 1994, for reviews), where convective plumes interact with the layer of shear. Most of the previous work on dynamo action in the tachocline has not sought to resolve the fluid motions in any detail, but has used a parameterized $\alpha$-effect, eddy diffusivity, and differential rotation. Typically the shear is large and the eddy diffusivity low within the tachocline, while just above there is no shear, but the $\alpha$-effect and eddy diffusivity are large. Models based on these concepts have exhibited kinematic and nonlinear dynamo waves and reproduced features of butterfly diagrams (Deluca \& Gilman 1986, 1988; Parker 1993; Prautzsch 1993; Rüdiger \& Brandenburg 1995; Tobias 1995; Roald 1998).

The problem of how to parameterize the $\alpha$-effect and eddy diffusion is avoided by the direct numerical simulation of the full governing equations, a computationally intensive task taken up by Brandenburg et al. (1990) and Nordlund et al. (1992). They studied flows in which convection overshoots into a stably stratified region, achieved by the enhancement of the local thermal diffusivity (Hurlburt, Toomre \& Massaguer 1986). They found dynamo action, with magnetic field largely concentrated at the interface between stable and unstable regions. Dynamo waves have also been obtained in models of turbulent accretion disks (Brandenburg et al. 1995); here motions are dominated by shear from the Keplerian orbits. These studies do not combine the effects of thermally driven convection and a layer of shear, unlike the work we shall present, but there are some large-scale simulations involving both of these effects in progress (Brandenburg, personal communication).

Although our study is broadly motivated by the problem of the Solar dynamo, we investigate basic flow configurations of a classical nature. The principal effects included in our simplified model are shear, rotation and convection. These are the building blocks of many astrophysical and geophysical flows (e.g. Zeldovich, Ruzmaikin \& Sokoloff 1983), and also occur in several projects aimed at exhibiting laboratory dynamos (Odier, Pinton \& Fauve 1998; Cawthorne, Peffley \& Lathrop 2000; Gailitis et al. 2000). In this paper we study kinematic dynamo action in a model of convection over a layer of shear. Our aim is to re- 
solve detailed fluid motions and so study dynamo action without introducing a parameterized $\alpha$-effect. Nevertheless, in order to explore the mathematically interesting and astrophysically important limit of large magnetic Reynolds number (see Childress \& Gilbert 1995), we limit our attention to two-dimensional fluid motions. Here the term two-dimensional refers to the dependence of the flow on just two space coordinates, though the motion itself has a three-dimensional character, having all three components, as is necessary to escape the consequences of the anti-toroidal dynamo theorem. We find that the nature of the resulting dynamo mechanism depends on the form of the motion, itself dependent on the instability mechanism which generates it. Of interest are the structure and growth rates of the magnetic fields generated and the extent to which they are localized in space.

The study of dynamo action in rotating convection has a long history and has developed along two distinct tracks stemming from the pioneering studies of Childress \& Soward (1972) and Busse (1975). They focused on the low magnetic Reynolds limit, for which an $\alpha$-effect can be determined robustly, that is, as an asymptotic result which is not the consequence of an unjustifiable mean field approximation. Magnetic field generation in the former plane layer model of Childress \& Soward (1972) relies heavily on the three-dimensional planform of the convective rolls in the weakly nonlinear regime. Subsequent developments have been discussed by Jones \& Roberts (2000), who do fully hydrodynamic three-dimensional simulations well into the nonlinear regime. Nevertheless, Matthews (1998) has shown recently that kinematic dynamo action is possible with finite-amplitude two-dimensional convection. Our work builds extensions into Matthews' basic model, the most important of which is shear flow, and addresses kinematic dynamo action in the limit of large magnetic Reynolds number.

The Busse (1975) model is rather different. Its pivotal idea is that the convection pattern in a rotating thick shell is quasi-geostrophic and, being largely independent of the coordinate parallel to the rotation axis, takes on the form of a cartridge belt. This led to his annulus model, in which gravity is radial and rotation $\boldsymbol{\Omega}$ is axial. A small surface inclination mimics the shell geometry. This slightly breaks the twodimensionality of the convective rolls, causing them to propagate like Rossby waves. The nonlinear development of such thermal Rossby waves has been investigated extensively by Busse and his co-workers. Kim, Hughes \& Soward (1999) have studied large magnetic Reynolds number dynamo action in the annulus configuration and they provide pertinent references to the earlier literature on non-magnetic convection studies. They were interested in the possibility of fast dynamo action in these models. Since this requires the existence of chaotic particle paths, their strategy was to investigate flows vigorous enough for that to occur. When their two-dimensional flow is sufficiently time-dependent, they show that there are regions of Lagrangian chaos, and obtain evidence for fast dynamo amplification of magnetic fields through the stretch-fold-shear mechanism. Since numerical results for the extremely high electrical conductivity appropriate to solar dynamos are unattainable, they obtained instead numerical results for various large values of the conductivity in order to determine the trend appropriate to the high conductivity limit. We adopt the same strategy but, whereas they consider the trend with increasing magnetic Prandtl number $v / \eta$, we increase the Roberts number $\kappa / \eta$.

Our paper is organized as follows. In $\S 2$ we set up our simplified model which captures the essential dynamical features of the tachocline and the lower reaches of the convection zone rotating differentially above it. Local Cartesian coordinates are 
adopted to describe the shell geometry at co-latitude $\vartheta$. To that end we consider an unbounded horizontal plane layer depth $h$ of Boussinesq fluid, which rotates rapidly about an axis inclined at an angle $\vartheta$ to the vertical. An adverse temperature gradient is maintained by heating from below, which when sufficiently strong drives thermal convection. In order to filter out the large-scale convective cells that cross the entire convection zone, we place the upper boundary of our model relatively close to its base at a height, which is determined by the scale of the small convective eddies believed responsible for the $\alpha$-effect in mean-field models.

The nature of the tachocline is such that there is strong local shear between the radiative interior and the convection zone, whose local bulk moves azimuthally at a different speed $U_{0}$. We emphasize that the axes for our local model are taken in the frame moving with the mean (i.e. bulk) motion of the convection zone, relative to which the radiative zone moves azimuthally backwards (forwards) at equatorial (polar) latitudes. To formulate a flow consistent with the physical picture, we apply no-slip conditions at the lower boundary which moves at constant velocity. Since the upper boundary is stationary in the co-moving frame, motion is insensitive to the stress boundary conditions on it and so again, for simplicity, we simply apply no-slip conditions. Consistent with the picture developed, we must demand that there is no horizontal pressure gradient in the local moving frame. (Note that there is a horizontal pressure gradient in the fixed frame rotating with the radiative interior; the pressure gradient is not Galilean invariant in rotating reference frames.) The effect is to generate a Couette-Ekman flow, which in the limit of strong vertical rotation becomes a thin Ekman layer localized near the bottom boundary, the flow being zero elsewhere. We remark that a consequence of our modelling is that, in addition to vertical azimuthal shear, the Ekman layer carries a north-south mass flux.

The magnitude of this north-south mass transport is of order $U_{0}(v / \Omega)^{1 / 2}$ and depends on the magnitude $U_{0}(\vartheta)$ of the differential rotation, which is latitude dependent. The ensuing Ekman pumping velocity is very small, of order $\left(U_{0} / L\right)(v / \Omega)^{1 / 2}$, where $L$ is the long north-south length scale of $U_{0}$. It leads to large-scale meridional circulations on the same length scale $L$. Nevertheless, most of this flux is returned high in the convective zone above the upper boundary height $h(\ll L)$ of our model. We are not concerned with these large-scale phenomena dependent on $L$, which are negligible on our small convective scale $h$.

In $\S 3$ we describe the numerical methods used to integrate the momentum, temperature and induction equations. Since, as already explained, all our motion is two-dimensional, the model may be pushed to high resolutions, enabling an accurate study of the large magnetic Reynolds number regime. In the absence of magnetic field, the basic state of flow and temperature gradient, which depends only on the vertical coordinate, may become unstable to both convective and hydrodynamical instabilities. In $\S 4$ we consider purely hydrodynamical instabilities of the Ekman layer flow at the base of the plane layer. These equilibrate in the form of rows of cat's eyes that translate steadily. We consider the dynamo effect of two of these flows, and find slow dynamo mechanisms which fall into two basic categories. We find cases where magnetic field growth is by the Ponomarenko dynamo mechanism on closed stream surfaces inside the cat's eyes. In other cases, growing fields are localized around hyperbolic stagnation points and their connecting separatrices, the G. O. Roberts (1972) mechanism. The simultaneous occurrence of both mechanisms in a fluid flow has recently also been found by Plunian, Marty \& Alemany (1999). 


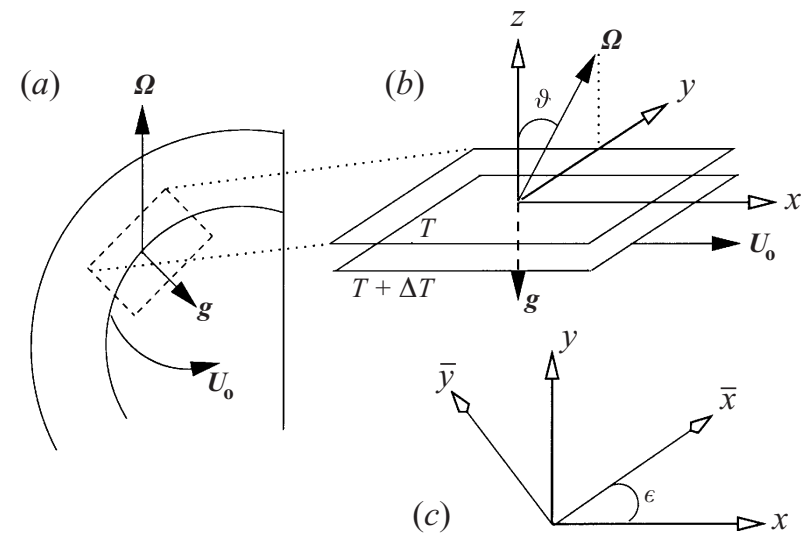

FiguRE 1. (a) Spherical geometry relevant to the Solar dynamo. The dashed box indicates schematically a local Cartesian approximation at a co-latitude $\vartheta$, which includes part of the convection zone and overshoot layer. $(b)$ This plane layer system is shown in more detail. The gravitational vector $\boldsymbol{g}$ is in the negative $z$-direction. The rotation vector $\boldsymbol{\Omega}$ lies in the $(y, z)$-plane, making an angle $\vartheta$ with the vertical. A velocity $\boldsymbol{U}_{0}=U_{0} \widehat{\boldsymbol{x}}$ in the $x$-direction is imposed at the base of the layer. The fluid layer is heated from below. $(c)$ The $(\bar{x}, \bar{y})$ axes are obtained from the $(x, y)$ axes by a rotation through $\epsilon$. The flow is independent of the $\bar{y}$-direction, which lies along the roll axes.

In $\S 5$ we consider parameter regimes in which the Ekman layer flow is hydrodynamically stable, and then introduce convective instability by increasing a Rayleigh number. Near to but above the onset of instability, the fluid flow is steady in a suitably chosen moving frame, and we recover similar dynamo mechanisms as discussed in $\S 4$ for the Ekman instability. Further from onset the flow gains additional time-dependence, becoming unsteady in any translating frame. At this point regions of Lagrangian trajectories become chaotic, initially near to the separatrices of the original steadily translating flow. Lagrangian chaos leads to exponential stretching of magnetic field lines and fast dynamo amplification in the large magnetic Reynolds number limit. Finally $\S 6$ offers some conclusions and discussion.

\section{Governing equations and invariants}

\subsection{Dimensionless equations}

We consider a local Cartesian approximation to spherical geometry, taking a layer of fluid of depth $h$ with $0 \leqslant z \leqslant h$, at co-latitude $\vartheta$, rotating with angular velocity $\boldsymbol{\Omega}$. In terms of a Solar dynamo, such a model would represent a region including the tachocline and only a part of the Solar convection zone, in which the effects of spherical geometry are negligible. The approximation is shown schematically in figure $1(a, b)$. We take Cartesian axes with $x$ directed eastwards, $y$ northwards and $z$ vertical, as shown. For definiteness we consider the layer to be located in the Northern hemisphere. The co-latitude then satisfies $0 \leqslant \vartheta \leqslant \pi / 2$ and with the rotation directed as shown:

$$
\boldsymbol{\Omega}=\Omega \widehat{\boldsymbol{\Omega}}=\Omega(\cos \vartheta \widehat{\boldsymbol{z}}+\sin \vartheta \widehat{\boldsymbol{y}}) \quad(\Omega \geqslant 0) .
$$

Our modelling of the tachocline is based on the idea that the convection zone differentially rotates above the radiative zone which itself rotates rigidly. Locally we adopt axes fixed in the local bulk zonal motion relative to which the rigid bottom 
boundary $z=0$ moves with velocity $\boldsymbol{U}_{0}=U_{0} \widehat{\boldsymbol{x}}$ dependent on the co-latitude $\vartheta$. Indeed, the sign of the shear in the tachocline changes with $\vartheta$; the case of $U_{0}<0(>0)$ is relevant for equatorial (polar) latitudes, where the convection zone super-rotates (subrotates) compared with the radiative interior. We assume that the differential rotation is geostrophic in character with the Coriolis acceleration balanced by a north-south pressure gradient. As explained in the Introduction, this means that, in our locally moving frame with no bulk motion, the horizontal pressure gradient, which is not Galilean invariant, vanishes.

To complete the specification of our model, the top boundary $z=h$ is maintained at a temperature $T$, while the lower hotter boundary $z=0$ is at temperature $T+\Delta T$. In order not to seriously disrupt the shear flow configuration described above, the upper rigid boundary is taken to be at rest. Thus provided the rotation vector is not parallel to the boundaries a strong Ekman layer forms on the bottom boundary with an inconsequential weak Ekman layer on the top; accordingly there is an up-down asymmetry which mimics the tachocline configuration. Instabilities of our flow are driven both thermally by the applied adverse temperature gradient and mechanically by the shear in the bottom Ekman layer, whose nature we discuss further below.

We will use the Boussinesq approximation. The fluid is taken to have a viscosity $v$, a thermal diffusivity $\kappa$, a magnetic diffusivity $\eta$, and a thermal coefficient of expansion $\alpha$. Length, time and velocity are non-dimensionalized using the depth $h$ and the thermal time scale $h^{2} / \kappa$. The equations for the velocity $\boldsymbol{U}$ of the fluid, the magnetic field $\boldsymbol{B}$, and the deviation $\theta$ of the temperature gradient from a steady conductive equilibrium, then take the standard form

$$
\begin{gathered}
\partial_{t} \boldsymbol{U}+\boldsymbol{U} \cdot \nabla \boldsymbol{U}+\tau P \widehat{\boldsymbol{\Omega}} \times \boldsymbol{U}=-\nabla \Pi+P \theta \widehat{\boldsymbol{z}}+P \nabla^{2} \boldsymbol{U}, \\
\partial_{t} \theta+\boldsymbol{U} \cdot \nabla \theta=R a U_{z}+\nabla^{2} \theta, \\
\partial_{t} \boldsymbol{B}+\boldsymbol{U} \cdot \nabla \boldsymbol{B}-\boldsymbol{B} \cdot \nabla \boldsymbol{U}=q^{-1} \nabla^{2} \boldsymbol{B}, \\
\nabla \cdot \boldsymbol{U}=\nabla \cdot \boldsymbol{B}=0,
\end{gathered}
$$

where the dimensionless parameters employed are

$$
R a=\frac{\alpha g h^{3} \Delta T}{v \kappa}, \quad \tau=\frac{2 \Omega h^{2}}{v}, \quad P=\frac{v}{\kappa}, \quad q=\frac{\kappa}{\eta},
$$

namely the Rayleigh, the square root of the Taylor, Prandtl and Roberts numbers respectively. The Lorentz force feedback is omitted in the momentum equation, as our present study is restricted to kinematic dynamo action.

Lacking vertical boundaries, we assume spatial periodicity in the unbounded horizontal direction. On the horizontal boundaries $z=0,1$, the temperature deviation $\theta=0$ and no-slip boundary conditions are used for the fluid. The magnetic field $\boldsymbol{B}$ is taken to obey insulating boundary conditions. Consequently $\boldsymbol{B}$ matches to an external vacuum field, which itself satisfies $\nabla \times \boldsymbol{B}=0$ throughout both the regions $z>1$ and $z<0$. As an alternative, perfectly conducting boundary conditions could have been reasonably adopted on one or both boundaries. However with these boundary conditions, the magnetic field tends to be trapped at the boundaries, the field is unable to be evacuated outside, and the generation of field is concentrated near the boundaries (see, for example, St. Pierre 1993). This can enhance dynamo action, and lower the critical magnetic Reynolds numbers significantly. We wanted to ensure that boundary conditions were not playing an important role in any dynamo we might observe numerically, and so employed the less favourable insulating boundary conditions. 
After non-dimensionalization, the velocity at the bottom boundary becomes

$$
\boldsymbol{U}=\frac{U_{0} h}{\kappa} \widehat{\boldsymbol{x}}=\operatorname{Re} P \widehat{\boldsymbol{x}} \quad(z=0),
$$

where we have introduced the Reynolds number

$$
R e=\frac{U_{0} h}{v} .
$$

Note that the Reynolds number Re defined here can take either sign, depending on the sign of $U_{0}$; however given that $\Omega$ is non-negative (see (2.1)), the square root of the Taylor number $\tau$ must be non-negative also.

The basic steady, non-magnetic, thermally conductive equilibrium state depends only on the vertical coordinate $z$. Though there is no temperature perturbation $\theta=0$, there is a shear flow

$$
\boldsymbol{U}=\operatorname{Re} P \boldsymbol{\Lambda}(z)=\operatorname{Re} P\left(\Lambda_{1}(z), \Lambda_{2}(z), 0\right),
$$

driven by the differential motion of the plane parallel boundaries. In the absence of an imposed horizontal pressure gradient, the horizontal components of $(2.2 a)$ are

$$
\partial_{z}^{2} \Lambda_{1}=-2 \mu^{2} \Lambda_{2}, \quad \partial_{z}^{2} \Lambda_{2}=2 \mu^{2} \Lambda_{1}, \quad \mu=\sqrt{\frac{1}{2} \tau \cos \vartheta} \geqslant 0,
$$

which must be solved subject to the non-slip boundary conditions

$$
\Lambda_{1}(0)=1, \quad \Lambda_{2}(0)=\Lambda_{1}(1)=\Lambda_{2}(1)=0 .
$$

The solution is

$$
\begin{aligned}
& \Lambda_{1}(z)=\frac{\cosh (\mu(z-2)) \cos (\mu z)-\cosh (\mu z) \cos (\mu(z-2))}{\cosh (2 \mu)-\cos (2 \mu)}, \\
& \Lambda_{2}(z)=\frac{\sinh (\mu(z-2)) \sin (\mu z)-\sinh (\mu z) \sin (\mu(z-2))}{\cosh (2 \mu)-\cos (2 \mu)}
\end{aligned}
$$

(see also Ponty, Gilbert \& Soward 2001), valid for $\mu>0$. In the limit $\mu \downarrow 0$ of no vertical rotation, we recover unidirectional plane Couette flow

$$
\Lambda_{1}(z)=1-z, \quad \Lambda_{2}(z)=0 \quad(\mu=0),
$$

while in the limit of strong vertical component of rotation, the flow

$$
\Lambda_{1} \sim \mathrm{e}^{-\mu z} \cos \mu z, \quad \Lambda_{2} \sim-\mathrm{e}^{-\mu z} \sin \mu z \quad(\mu \gg 1),
$$

is localized near the bottom boundary $z=0$, in the form of an Ekman layer of width order $\mu^{-1}$, which exhibits the characteristic Ekman spiral profile. Note particularly that our choice of non-symmetric boundary conditions $(2.8)$ on $\Lambda_{1}$ (together with the assumed zero horizontal pressure gradient) has the important effect of breaking the up-down symmetry in a non-trivial way. Indeed, the presence of just one Ekman layer in our system means that there is a mean horizontal flux of fluid. Were we to move both boundaries in opposite directions (say $\Lambda_{1}(0)=1$ and $\Lambda_{1}(1)=-1$ ), there would be no mean flux and we would regain up-down symmetry $\Lambda(1-z)=-\Lambda(z)$ with two Ekman layers, one at the top, the other at the bottom, as in the study of Hoffmann, Busse \& Chen (1998).

Once the full flow $\boldsymbol{U}$ deviates from the basic Ekman layer shear flow solution, it is convenient to write

$$
\boldsymbol{U}=\operatorname{Re} P \boldsymbol{\Lambda}(z)+\boldsymbol{u}
$$


Accordingly the non-slip boundary conditions imply that $\boldsymbol{u}=0$ at the top and bottom of the layer. With the substitution (2.12), the governing equations $(2.2 a-c)$ become

$$
\begin{aligned}
& \partial_{t} \boldsymbol{u}+\boldsymbol{u} \cdot \nabla \boldsymbol{u}+\operatorname{Re} P\left(\boldsymbol{\Lambda} \cdot \nabla \boldsymbol{u}+u_{z} \partial_{z} \boldsymbol{\Lambda}\right)+\tau P \widehat{\boldsymbol{\Omega}} \times \boldsymbol{u}=-\nabla \Pi+P \theta \widehat{\boldsymbol{z}}+P \nabla^{2} \boldsymbol{u}, \\
& \partial_{t} \theta+\boldsymbol{u} \cdot \nabla \theta+\operatorname{Re} P \boldsymbol{\Lambda} \cdot \nabla \theta=\operatorname{Ra}_{z}+\nabla^{2} \theta, \\
& \partial_{t} \boldsymbol{B}+\boldsymbol{u} \cdot \nabla \boldsymbol{B}-\boldsymbol{B} \cdot \nabla \boldsymbol{u}+\operatorname{Re} P\left(\boldsymbol{\Lambda} \cdot \nabla \boldsymbol{B}-B_{z} \partial_{z} \boldsymbol{\Lambda}\right)=q^{-1} \nabla^{2} \boldsymbol{B} .
\end{aligned}
$$

The above equations, together with the definitions of $\widehat{\boldsymbol{\Omega}}$ in (2.1) and $\boldsymbol{\Lambda}$ in (2.9), involve six independent parameters $\tau, P, R e, R a, \vartheta$ and $q$. They must be solved on $0<z<1$ subject to the non-slip, $\boldsymbol{u}=0$, and isothermal, $\theta=0$, boundary conditions on $z=0$ and $z=1$, together with the matching of $\boldsymbol{B}$ to an external potential magnetic field as mentioned in the paragraph following (2.3).

\subsection{Two-dimensional formulation}

The steady conductive equilibrium is dependent on the vertical coordinate $z$ but independent of the horizontal coordinates $x$ and $y$. Nevertheless, rotational invariance about the $z$-axis is broken by the presence of the horizontal component of rotation and the direction of the imposed motion of the bottom boundary. Thus at the onset of fluid instability, whether hydrodynamic or convective, the fluid motion takes the form of rolls with a specific orientation, and as result the flow is independent of a coordinate along the roll axes, which lie in the direction $(-\sin \epsilon, \cos \epsilon, 0)$, say. Of course, we assume here that there is no eigenvalue degeneracy, which could lead to the competition of rolls with different orientations such as in the Childress \& Soward (1972) dynamo. We therefore introduce new axes $(\bar{x}, \bar{y})$ rotated by an angle $\epsilon$ about the old axes (see figure $1 c$ ) so that $\bar{y}$ is directed along the roll axes and the flow $\boldsymbol{u}$ is a function of $\bar{x}, z$ and $t$ alone at onset. Relative to our new coordinates, we set

$$
\widehat{\boldsymbol{\Omega}}=\sin \vartheta \sin \epsilon \widehat{\overline{\boldsymbol{x}}}+\sin \vartheta \cos \epsilon \widehat{\overline{\boldsymbol{y}}}+\cos \vartheta \widehat{\boldsymbol{z}}
$$

and

$$
\boldsymbol{\Lambda}=\lambda_{1}(z) \widehat{\overline{\boldsymbol{x}}}+\lambda_{2}(z) \widehat{\overline{\boldsymbol{y}}}
$$

where

$$
\lambda_{1}=\Lambda_{1} \cos \epsilon+\Lambda_{2} \sin \epsilon, \quad \lambda_{2}=-\Lambda_{1} \sin \epsilon+\Lambda_{2} \cos \epsilon .
$$

Though the two-dimensionality of the flow at onset is clear, it is important to appreciate that we assume that the flow continues to take this restricted form $\boldsymbol{u}(\bar{x}, z, t)$ in the fully developed nonlinear state. Such a representation is completely consistent with the nonlinear governing equations (2.13), and allows the dynamo generation of three-dimensional magnetic field in the kinematic regime, when we ignore the feedback by the Lorenz force. We do not consider the possibility that following instability the fluid flow may become fully three-dimensional with structure along the roll axes. Our simplifying assumption of two-dimensionality, however, is important because it allows us to study numerically kinematic dynamo action at large magnetic Reynolds number.

Since $\boldsymbol{u}$ is independent of $\bar{y}$, we may introduce a stream function $\psi$ and write

$$
\boldsymbol{u}=-\partial_{z} \psi \widehat{\overline{\boldsymbol{x}}}+v \hat{\overline{\boldsymbol{y}}}+\partial_{\bar{x}} \psi \widehat{\boldsymbol{z}}=\nabla \times(\psi \widehat{\overline{\boldsymbol{y}}})+v \hat{\overline{\boldsymbol{y}}} .
$$

After substitution into (2.13) and upon taking $\widehat{\overline{\boldsymbol{y}}} \cdot(2.13 a), \widehat{\overline{\boldsymbol{y}}} \cdot \nabla \times(2.13 a)$ and $(2.13 b)$, 
we obtain a nonlinear matrix system for the $\psi, v$ and $\theta$ fields:

$$
\left(\partial_{t}+\operatorname{Re} P \lambda_{1} \partial_{\bar{x}}\right) \mathscr{W} X=\mathscr{B} \mathscr{X}+\operatorname{Re}\left(\begin{array}{l}
\lambda_{1}^{\prime \prime} \\
-\lambda_{2}^{\prime} \\
0
\end{array}\right) \partial_{\bar{x}} \psi-\frac{\partial(\psi, \mathscr{W} \mathscr{X})}{\partial(\bar{x}, z)},
$$

where

$$
\mathscr{X}=\left(\begin{array}{c}
\psi \\
v \\
\theta
\end{array}\right), \quad \mathscr{W}=\left(\begin{array}{ccc}
P^{-1} \Delta & 0 & 0 \\
0 & P^{-1} & 0 \\
0 & 0 & 1
\end{array}\right), \quad \mathscr{B}=\left(\begin{array}{ccc}
\Delta^{2} & -\tau \mathscr{D} & \partial_{\bar{x}} \\
\tau \mathscr{D} & \Delta & 0 \\
R a \partial_{\bar{x}} & 0 & \Delta
\end{array}\right),
$$

in which we have introduced the differential operators

$$
\mathscr{D}=(\widehat{\boldsymbol{\Omega}} \cdot \nabla)=\cos \vartheta \partial_{z}+\sin \vartheta \sin \epsilon \partial_{\bar{x}}, \quad \Delta=\partial_{\bar{x}}^{2}+\partial_{z}^{2},
$$

the latter being the horizontal Laplacian. For the case of no imposed shear flow, $R e=0$, the above matrix formulation is the standard representation for convection in a rotating Boussinesq fluid. The terms proportional to $R e$ on the left-hand side represent horizontal advection by the shear flow, while those on the right-hand side describe vertical advection of the mean shear flow by the velocity component $u_{z}=\partial_{\bar{x}} \psi$.

To investigate the possibility of kinematic dynamo action in our $\bar{y}$-independent fluid flow, we consider a magnetic field described by $\bar{y}$-dependent normal modes of the form

$$
\boldsymbol{B}=\boldsymbol{b}(\bar{x}, z, t) \exp (\mathrm{i} l \bar{y}) \quad(l \neq 0),
$$

with non-zero wavenumber $l$ necessary for dynamo action. The equations for $b_{\bar{x}}$ and $b_{z}$ may be found by substituting (2.12) and (2.21) into (2.13c). They are

$$
\begin{array}{r}
\partial_{t} b_{\bar{x}}=-\left[-\left(\partial_{z} \psi\right)\left(\partial_{\bar{x}} b_{\bar{x}}\right)+\mathrm{i} l v b_{\bar{x}}+\left(\partial_{\bar{x}} \psi\right)\left(\partial_{z} b_{\bar{x}}\right)+\left(\partial_{\bar{x} z} \psi\right) b_{\bar{x}}+\left(\partial_{z z} \psi\right) b_{z}\right] \\
-\operatorname{Re} P\left[\lambda_{1} \partial_{\bar{x}} b_{\bar{x}}+\mathrm{i} l \lambda_{2} b_{\bar{x}}-\lambda_{1}^{\prime} b_{z}\right]+q^{-1}\left(\partial_{\bar{x} \bar{x}}+\partial_{z z}-l^{2}\right) b_{\bar{x}}, \\
\partial_{t} b_{z}=-\left[-\left(\partial_{z} \psi\right)\left(\partial_{\bar{x}} b_{z}\right)+\mathrm{i} l v b_{z}+\left(\partial_{\bar{x}} \psi\right)\left(\partial_{z} b_{z}\right)-\left(\partial_{\bar{x} z} \psi\right) b_{z}-\left(\partial_{\bar{x} \bar{x}} \psi\right) b_{\bar{x}}\right] \\
-\operatorname{Re} P\left[\lambda_{1} \partial_{\bar{x}} b_{z}+\mathrm{i} l \lambda_{2} b_{z}\right]+q^{-1}\left(\partial_{\bar{x} \bar{x}}+\partial_{z z}-l^{2}\right) b_{z} .
\end{array}
$$

The remaining component $b_{\bar{y}}$ is determined directly by the solenoidal condition $(2.13 d)$ :

$$
-\mathrm{i} l b_{\bar{y}}=\partial_{\bar{x}} b_{\bar{x}}+\partial_{z} b_{z} .
$$

We remark that there appears to be no particular advantage in using a toroidalpoloidal decomposition for the magnetic field in our study.

\section{Numerical method and diagnostics}

The solution of the hydrodynamic system (2.18) and the induction equation is achieved numerically using a time-stepping pseudo-spectral code. The fields $\left(\psi, v, \theta, b_{\bar{x}}, b_{z}\right)$ are functions of $(\bar{x}, z, t)$ alone. In the vertical they are resolved using a 
Chebychev polynomial collocation method. In the horizontal $\bar{x}$-direction all fields are assumed to be periodic with periodicity $L=2 \pi / k_{c}$, where $k_{c}$ is the preferred wavenumber for linear hydrodynamic or convective instability at onset. Fourier modes are used to resolve the field in the $\bar{x}$-direction.

The non-slip boundary condition for the flow and the vacuum insulating boundary condition for the magnetic field are implemented with the tau method (see Gottlieb \& Orszag 1977). Typical resolutions are 128-256 collocation points in the horizontal and 65-129 in the vertical. The time-stepping applies the Crank-Nicholson method for the diffusion terms, and the Adams-Bashford method for the remaining terms. As tests of major parts of the code, it was verified that thresholds for the onset of Ekman and convective instabilities are in agreement with results obtained by an eigenvalue code (Ponty et al. 2001). Furthermore growth rates obtained with this code for kinematic dynamos of Ponomarenko type are in good agreement with asymptotic analysis (Gilbert \& Ponty 2000).

Our numerical study proceeds as follows. First we fix the parameters $\vartheta, P$ and $\tau$; in fact $P=1$ for all the runs described in this paper. This fixes the geometry and rotation, and a basic conducting state independent of $z$. Then mechanical or thermal forcing is introduced, and at some critical value of $R e$ or $R a$, the layer becomes unstable to rolls with wavenumber $k_{c}$ and axes at angle $\epsilon$ to the $y$-axis. This enables us to fix the location of the $(\bar{x}, \bar{y})$ axes and the length of the periodicity box in the $\bar{x}$-direction, $L=2 \pi / k_{c}$. This linear problem is addressed in the companion paper (Ponty et al. 2001).

Now keeping the other parameters fixed, and keeping the flow two-dimensional, the forcing given by $R e$ or $R a$ is increased and the branch of saturated hydrodynamic or convective instability is followed. At a given point along the branch we have an equilibrated flow, which could be steady or time-dependent. This flow may then be tested for dynamo action by introducing a magnetic field. For each value of the magnetic wavenumber $l$ a real dynamo growth rate $\sigma$ may be obtained as a function of the Roberts number $q$. In the high conductivity limit, both the Roberts number and magnetic Reynolds number are large and the primary concern is the distinction between fast and slow dynamos, and the mechanisms which support them.

To understand the results of our simulations, the most important parameter from the kinematic dynamo viewpoint is the magnetic Reynolds number. Since the flow may be driven mechanically through the motion of the bottom boundary, $R e \neq 0$, or through heating, $R a>0$, it is best to define the magnetic Reynolds number in terms of the flow as it is actually realized (rather than use $U_{0}$ to form the dimensionless combination of input parameters $\left.U_{0} h / \eta=\operatorname{Re} P q\right)$. Thus we introduce $U$ as the rootmean-square value of the non-dimensional total velocity $\boldsymbol{U}$ (see (2.12)), averaged over both space and time, and define the magnetic Reynolds number by

$$
R m=q U .
$$

We stress that this is a quantity measured from the simulations, unlike the dimensionless quantities defined in (2.3). Also, since the real magnetic field growth rate $\sigma$ is measured on the thermal diffusion time scale, our understanding the behaviour in the large $R m$ limit is helped by the introduction of the rescaled growth rate

$$
\lambda=\sigma / U
$$

based on the turnover time scale. Thus the dynamo is fast when $\lambda=O(1)$ in the limit $R m \rightarrow \infty$. 
Kinematic dynamo action in large magnetic Reynolds number flows

\section{Ekman instability branch}

\subsection{Hydrodynamic behaviour}

In the absence of any thermal driving, $R a=0$, the Ekman layer in our model becomes hydrodynamically unstable at sufficiently large Reynolds number Re. In this section we study the resulting flows and their kinematic dynamo properties.

For the case of purely vertical rotation $(\vartheta=0)$, the Ekman layer instability has been studied experimentally by Faller (1963) and Caldwell \& Van Atta (1970), and numerically by Faller \& Kaylor (1966), Lilly (1966), Iooss, Nielsen \& True (1978) and Melander (1983). The linear and nonlinear behaviour of Ekman-Couette instabilities in a plane layer for a wide range of rotation rates has been discussed by Hoffmann et al. (1998). The presence of the horizontal component of rotation $(0<\vartheta<\pi / 2)$ has an important effect on the Ekman instability, as shown by Leibovich \& Lele (1985). With our definition of $U_{0}$ and the Reynolds number $R e$ (see (2.5)), increasing horizontal rotation at fixed Taylor number $\tau^{2}$ by varying the co-latitude $\vartheta$ causes the magnitude $\left|R e_{c}\right|$ of the critical Reynolds number for the Ekman instability to decrease (increase) for positive (negative) $R e$ (see Ponty et al. 2001). As in the TaylorCouette instability, when the vorticity associated with the shear across the layer is aligned (anti-aligned) with the horizontal rotation vector, the effect is stabilizing (destabilizing). The transition between the Taylor-Couette instability and the Ekman layer instability is explored in Hoffman \& Busse (1999).

Two different Ekman layer instabilities are distinguished in these studies, which for historical reasons are now referred to as types I and II. Type II occurs when the Reynolds number $R e^{*}=R e / \mu$ defined using the Ekman layer thickness, which is more relevant than the depth of the layer, exceeds the experimentally measured value of $R e_{c}^{*} \simeq 56.7$ (or 124.5 for type I); the instability is manifest as a travelling wave having a wavelength of approximately $22 \mu^{-1}$ (or $11 \mu^{-1}$ for type I). We will focus on the type II travelling wave, which has the smaller critical Reynolds number and so is easier to study numerically.

We simulated the finite-amplitude development of the Ekman instability with our nonlinear numerical code subject to the two-dimensional restrictions already mentioned. Within that framework, we reach Reynolds numbers of up to 800 and find that the saturated flow remains steady in a frame moving with the phase velocity $U_{\text {roll }}$ of the roll in the $\bar{x}$-direction; this velocity is roll-amplitude dependent and changes as the solution branch is followed by varying the Reynolds number. It is plausible that the next bifurcation will involve the destabilization of modes with wavenumber components along the $\bar{y}$-axis, which are not present in our study; we will not pursue that possibility. Since the flow is steady in a moving frame, it cannot be chaotic and so any dynamo cannot be fast (see Klapper \& Young (1995) for rigorous results). Nevertheless, we have found robust slow dynamo action, as we now discuss.

\subsection{Slow dynamo action}

We consider two examples of dynamo action in the flow resulting from the equilibrated Ekman layer instability. In our examples, the critical Reynolds number and the orientation of the fluid rolls have been calculated from a linear stability code. In the two cases, the co-latitude is taken as $\vartheta=45^{\circ}$ (see $\S 4.2 .1$ ) and $67.5^{\circ}$ (see $\S 4.2 .2$ ) with a positive value of the Reynolds number. The first example is near the onset of the instability, and in the second example, the Reynolds number is around twice the critical value.

In both these cases the rolls are steady in a frame moving with velocity $U_{\text {roll }}$ along 
the $\bar{x}$-axis, which must be measured from the simulation. From the simulation a quantity, such as kinetic energy integrated over a periodicity box, remains constant, while a signal such as a velocity component at a given point oscillates periodically, with period $2 \pi / \omega$. If a power spectrum of the signal is taken, the lowest peak is at the frequency $\omega$, the remaining peaks being multiples of $\omega$. This allows us to measure $U_{\text {roll }}=\omega / k_{c}$. It is then helpful to define a total stream function $\Psi$ for the flow in the moving frame by

$$
\Psi(\bar{x}, z)=\psi(\bar{x}, z)-\operatorname{Re} P \int_{0}^{z} \lambda_{1}(\xi) \mathrm{d} \xi+U_{\text {roll }} z
$$

so that the $\bar{x}$ and $z$ components of the total velocity in this frame are

$$
U-U_{\text {roll }}=-\partial_{z} \Psi, \quad W=\partial_{\bar{x}} \Psi .
$$

The total velocity $V$ along the $\bar{y}$-axis is also defined by

$$
V(\bar{x}, z)=v(\bar{x}, z)+\operatorname{Re} P \lambda_{2}(z) .
$$

The total stream function $\Psi$ is very useful, because in the frame co-moving with the rolls, particles follow the stream lines $\Psi=$ const. In the large- $R m$ limit magnetic field lines tend to align with the steady flow, and the dynamo-generated field tends to localize in the vicinity of particular stream surfaces.

\subsubsection{First example: $\vartheta=45^{\circ}$}

For our first example, we take $\vartheta=45^{\circ}, \tau=60, \epsilon=73.5^{\circ}, k_{c}=3.44$ and $R e=210$ (the critical value being $R e_{c} \simeq 190$ ). The results are illustrated in figure $2(a, b)$, which gives the $\bar{y}$-velocity $V$ and total stream function $\Psi$ in the co-moving frame. The flow has a cat's eye configuration typical of finite-amplitude instability, which is manifest at critical levels in other shear flows, e.g. the Kelvin-Helmholtz instability. In the $(\bar{x}, z)$ plane, this streamline topology contains elliptic and hyperbolic stagnation points; the latter are joined by heteroclinic connections, or separatrices. The exponential stretching at the stagnation points, and the differential rotation around the elliptic points, can stretch out field, contributing to a dynamo process. The velocity component $V$ in the $\bar{y}$-direction shown in figure $2(a)$, which results from the overall rotation of the system, is however crucial for dynamo action. The single dark line contour on figure $2(a)$ shows where the sign of $V$ changes. Also visible in figure $2(a, b)$ is the intense shear of the Ekman layer at the base of the plane layer.

Figure 2(c) shows the magnetic field for the mode $l=1.2$ with $q=50$; the corresponding magnetic Reynolds number for the resulting flow is measured to be $R m \simeq 3660$. The magnetic field plotted is the root-mean-square value,

$$
B=\left(\left|b_{\bar{x}}\right|^{2}+\left|b_{\bar{y}}\right|^{2}+\left|b_{z}\right|^{2}\right)^{1 / 2},
$$

which is averaged over $\bar{y}$, but then normalized so as to lie between zero and one. This collapses all the structure in the $\bar{y}$-direction onto the $(\bar{x}, z)$-plane.

Figure 3 shows the magnetic growth rate as a function of the wavenumber $l$ for the same flow and the same magnetic Reynolds number employed in figure 2 . The growth rate is shown on the left-hand scale as $\sigma$, based on the thermal diffusion time, and on the right-hand scale as $\lambda$, based on the turnover time. The graph has a positive maximum at $l=1.2$, giving the dominant mode shown in figure $2(c)$; note also that large-scale fields with small values of $l$ possess positive growth rate.

Comparing figures $2(b)$ and $2(c)$ we see that the dominant magnetic mode has field localized in sheets along the separatrices of the flow in the $(\bar{x}, z)$-plane (using 

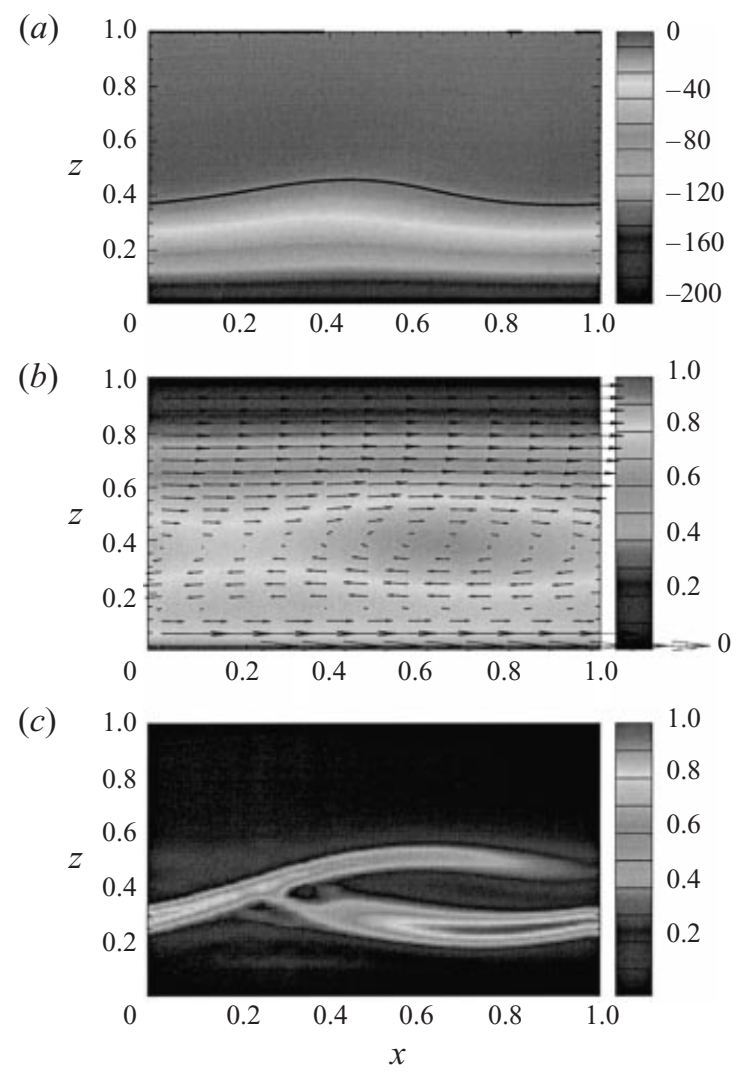

FIGURE 2. A flow resulting from a saturated Ekman layer instability drives a dynamo for $\vartheta=45^{\circ}$, $\tau=60, \epsilon=73.5^{\circ}, k_{c}=3.44, R e=210$ and $q=50$, corresponding to $R m \simeq 3660$, with the wavenumber $l=1.2$. It is shown as a time-snapshot in the $(\bar{x}, z)$-plane of $(a)$ the total velocity $V$ along the $\bar{y}$-axis (the dark contour line shows the change in sign of $V$ ), $(b)$ the total stream function $\Psi$ with arrows showing the velocity vectors, and $(c)$ the magnitude $B$ of the magnetic field.

the stream function $\Psi$ for the appropriate moving frame). The sheets intersect at a hyperbolic stagnation point. Our simulation represents the first example of a dynamo effect obtained in a cat's eyes configuration resulting from hydrodynamic instability. The dynamo mechanism has some similarities with that of the periodic G. O. Roberts (1972) cellular flow investigated analytically in the large- $R m$ limit by Childress (1979) and Soward (1987). In this case the field is also associated with the stagnation points and separatrices, and is amplified by a steady stretch-fold-shear mechanism. The dynamo is slow (unless the flow field is modified so as to be discontinuous) but growth rates fall off extremely slowly as $R m$ is increased. Dynamo action is also considered for doubly periodic rows of cat's eyes by Childress \& Soward (1989), who compute an $\alpha$-effect associated with boundary layers at separatrices. Their calculations involve averaging over the infinite plane, and it remains to be clarified as to how their results relate to dynamo action in a row of cat's eyes in a plane layer with insulating boundaries, as in our model.

\subsubsection{Second example: $\vartheta=67.5^{\circ}$}

In the second example of dynamo action in flows resulting from the Ekman instability, we take $\vartheta=67.5^{\circ}, \tau=100, \epsilon=79.28^{\circ}$ and $k_{c}=4.30$. The Reynolds 


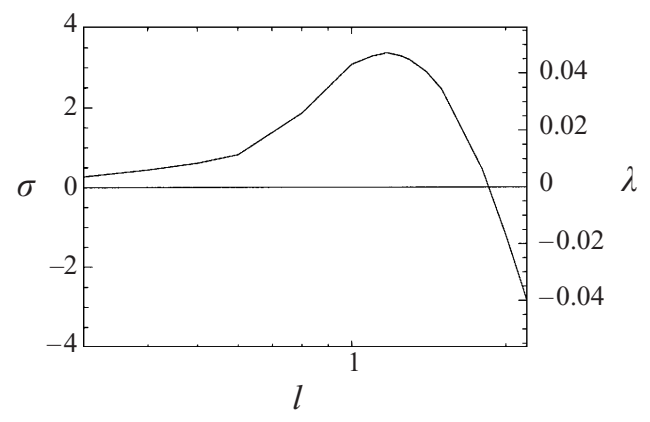

FIGURE 3. The magnetic field growth rate plotted against the wavenumber $l$ along the $\bar{y}$-axis, with two scalings: $\sigma$ on the left-hand side is scaled with the thermal diffusion time, and $\lambda$ on the right-hand side with the turnover time. The flow is an equilibrated Ekman layer instability with parameters as in figure 2 .

number is $R e=250$, which is to be compared to the critical value for the instability of $R e_{c} \simeq 138$. Again the resulting flow is steady in a moving frame, and figures $4(a)$ and $4(b)$ show respectively the $\bar{y}$-velocity and the stream function $\Psi$ in the moving frame. The flow is now more complicated than that in figure 2, with two eddies per periodicity length, one rotating clockwise, and another counter-rotating (across the horizontal box boundary).

This example exhibits different dynamo mechanisms depending on the wavenumber $l$. Figure 5 shows the growth rates $\sigma$ and $\lambda$ plotted against the wavenumber $l$ for $q=40$, which corresponds to $R m \simeq 3600$. The curve is complicated, having many peaks and windows of dynamo and non-dynamo action as $l$ is varied. Below the wavenumber $l \simeq 2.0$, the magnetic field shown in figure $4(c)$ (with $l=1.2$ ) is concentrated around the principal vortices of the flow displayed in figure $4(b)$. The dynamo process appears to be that in the Ponomarenko dynamo (Ponomarenko 1973; Gilbert 1988; Ruzmaikin, Sokoloff \& Shukurov 1988; Gilbert \& Ponty 2000). Field directed in or out of the eddy is stretched by differential rotation in helical streamlines to generate field directed along the streamlines. Diffusion of this field in curved geometry generates field across streamlines and closes the dynamo loop, leading to the amplification of magnetic field. To confirm this picture the magnetic field is visualized in three dimensions in figure 6 and takes the form of two spiralling tubes. Above the wavenumber $l \simeq 2.0$, the magnetic field is localized along the separatrices, and corresponding stagnation points, as in the previous case (figure 2). The magnitude of the magnetic field is shown in figure $4(d)$ for $l=9.0$, where the magnetic growth rate is a maximum in figure 5 .

In this example of a saturated Ekman instability, we have identified two different dynamo mechanisms which compete with each other. The stretch-fold-shear mechanism in the steady flow is the more efficient one, preferring modes with short scale in the $\bar{y}$-direction, but the Ponomarenko mechanism is also there, subdominant, preferring large-scale fields. Note that regardless of the wavenumber $l$ in the $\bar{y}$-direction the two mechanisms tend to amplify fields of different length scales in the $(\bar{x}, z)$-plane for large $R m$, as seen in figure $4(c, d)$. For $l=O(1)$ the Ponomarenko dynamo amplifies field on an $O\left(R m^{-1 / 4}\right)$ length scale (see Gilbert \& Ponty 2000), while the fields associated with the hyperbolic stagnation points localize on an $O\left(\mathrm{Rm}^{-1 / 2}\right)$ length scale (Childress 1979), which is rather more testing numerically. 
Kinematic dynamo action in large magnetic Reynolds number flows

(a)

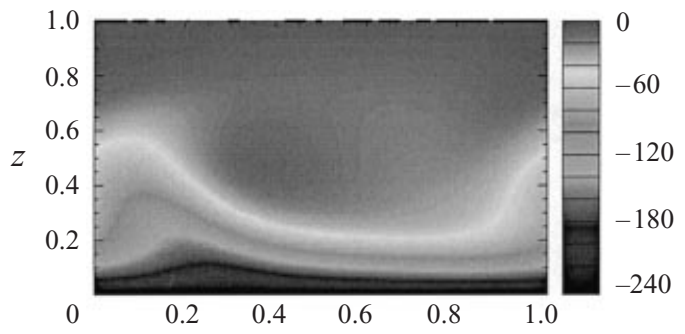

(b)

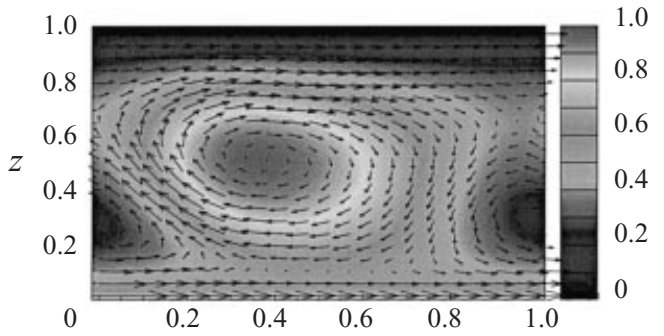

(c)

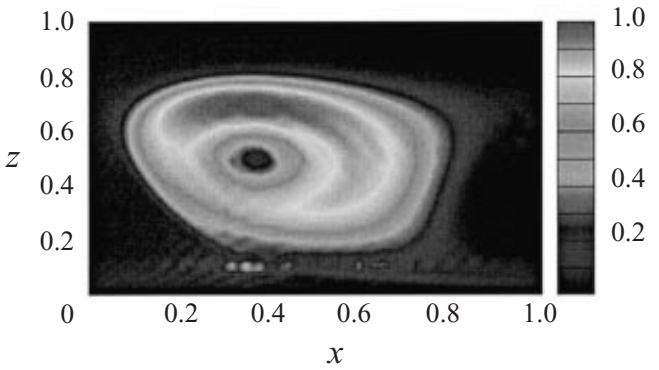

(d)

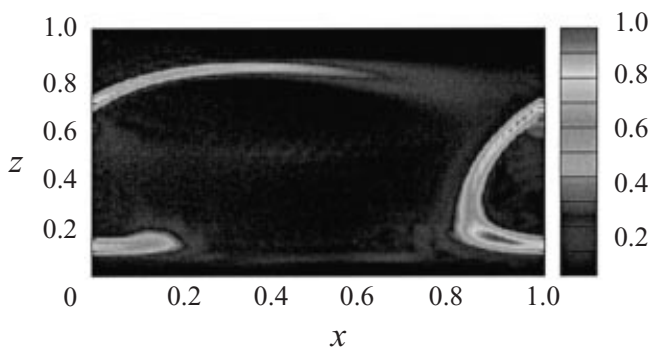

FIgURE 4. A flow resulting from a saturated Ekman layer instability drives a dynamo for $\vartheta=67.5^{\circ}$, $\tau=100, \epsilon=79.28^{\circ}, k_{c}=4.64, R e=250$ and $q=40$, corresponding to $R m \simeq 3600$. It is shown as a time-snapshot in the $(\bar{x}, z)$-plane of $(a)$ the total velocity $V$ along the $\bar{y}$-axis, $(b)$ the total stream function $\Psi,(c)$ the magnitude $B$ of the magnetic field in the case $l=1.2$, and $(d)$ the magnitude $B$ of the magnetic field in the case $l=9.0$.

\section{Convective branch}

\subsection{Behaviour of convection}

In this section the Reynolds numbers involved are sufficiently low that the purely hydrodynamical Ekman instability is not present. The flow is then destabilized by heating from below, $R a>0$, leading to thermal instability. To illustrate our results, two cases are presented: the first one, discussed in $\$ \S 5.2$ and 5.3, has the vertical rotation orientated with an angle $\vartheta=67.5^{\circ}$, and the second, discussed in $\S 5.4$, has a 


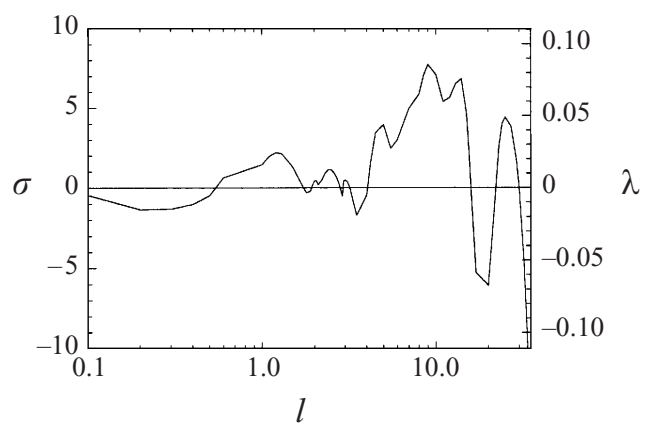

FIGURE 5. The magnetic field growth rate plotted against the wavenumber $l$ along the $\bar{y}$-axis, with two scalings: $\sigma$ on the left-hand side is scaled with the thermal diffusion time, and $\lambda$ on the right-hand side with the turnover time. The flow is an equilibrated Ekman layer instability with parameters as in figure 4.

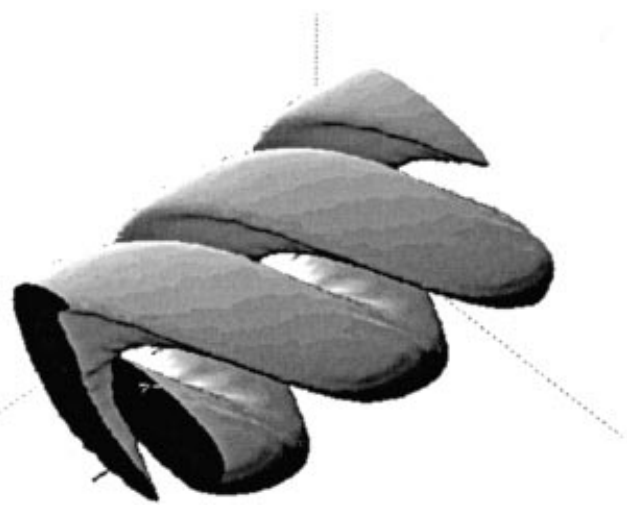

FIGURE 6. A three-dimensional visualization of the magnetic field in the case shown in figure 4(c).

An iso-surface of constant magnitude of magnetic field is plotted with respect to $(\bar{x}, \bar{y}, z)$ axes.

purely vertical rotation vector, $\vartheta=0^{\circ}$. In both examples the Prandtl number is equal to one, so that viscous and thermal time scales are the same.

At the onset of the instability, convective rolls appear. The presence of a horizontal component of rotation $(\vartheta \neq 0)$ or of a shear flow $R e \neq 0$ breaks the symmetry of horizontal isotropy and gives a preferred orientation to the convective rolls. When the rotation is purely horizontal $\vartheta=\pi / 2$ and the shear is present in the form of a linear Couette flow (2.10), there is a competition between their physical effects, but the orientation of the rolls remains simple: their axes are either aligned with the shear, or with the horizontal component of rotation (Kropp \& Busse 1991; Matthews \& Cox 1997). However our case also includes a vertical component of rotation, and this together with the shear leads to a spiral Ekman layer flow (2.9) at the base of the layer. With a spiral Ekman layer, there are no simple results giving the orientation of convective rolls at onset, and the preferred orientation has to be calculated numerically or asymptotically (Ponty et al. 2001).

All the parameters of the convection threshold are then calculated with a numerical code solving the linear stability problem. The parameter $\epsilon$, giving the orientation of the coordinate system in both cases, is fixed by the value preferred at onset, and similarly for $k_{c}$ which fixes the box dimension. The parameter regime taken in our first case was motivated by the fact that the roll axes are quite close to the north- 
south orientation. Indeed, the effect of the Coriolis force on a rapidly rotating fluid body tends to produce convection in the form of travelling waves whose axes have a preferential north-south orientation in accord with the Taylor-Proudman constraint. The classic picture shows columnar convection rolls or travelling waves localized at a specific radius of a sphere and orientated north-south (Busse 1970, 1994).

\subsection{Slow and fast dynamo action for $\vartheta=67.5^{\circ}$}

Our first example involves all the physical ingredients present in our system, and so represents the general case. We consider $\vartheta=67.5^{\circ}, \tau=200$ and $R e=30$ (we obtain qualitatively similar results for negative values of $R e$ ). For these values thermal instability occurs at $R a_{c} \simeq 3748$, with corresponding wavenumber given by $\epsilon=2.33^{\circ}$, $k_{c}=4.30$. Figure $7(a, b)$ shows the flow near to onset, with $R a=4125 \simeq 1.1 R a_{c}$. The flow is steady in a moving frame and figure $7(b)$ shows the stream function in this frame. The vertical velocity is produced by the convective motion, but the horizontal velocity is dominated by the Ekman mean flow, seen at the bottom of figure $7(a, b)$. Figure $7(c)$ shows the magnetic field with a wavenumber $l=8.5$ and a Roberts number $q=50$, corresponding to $R m \simeq 500$. Being steady in a suitable frame, the flow produces a slow kinematic dynamo mechanism and the magnetic field is localized along the separatrix near the base of the layer.

The fields generated by this dynamo mostly occur in and above the Ekman layer, where there is considerable shear, especially in the perpendicular velocity component $V$ (figure $7 a$ ). It appears that the shear in the Ekman layer is playing an important part in the amplification process, presumably by a steady stretch-fold-shear mechanism. Of course the weaker vertical motions arising from the convection are also needed. This mechanism seems robust in our simulations, being found in many parameter regimes. It would be interesting to model the dynamo analytically, as a generic mechanism in which an Ekman mean flow dominating the horizontal velocity is perturbed by weak vertical motions, driven by any kind of hydrodynamic or thermal instability.

When the Rayleigh number is increased further, holding other parameters constant, a bifurcation occurs (at approximately $18 R a_{c}$ ) and the flow in any moving frame becomes time-dependent; we refer to this as the flow having intrinsic time-dependence, that is, which cannot be removed by a Galilean transformation. After this bifurcation integrated quantities such as the kinetic energy show periodic time-dependence, and a signal such as a component of velocity at a given point is generally quasi-periodic. However, as one goes through the bifurcation it is possible to adjust $U_{\text {roll }}$ so that the flow is strictly periodic in a frame with this velocity. To do this note that if $U_{\text {roll }}$ is near to, but not equal to, the correct value, the signal from a given point in space will be quasi-periodic, with one particularly low frequency. This can be extracted from a power spectrum, and provides a better estimate of $U_{\text {roll }}$. Thus numerically we can find this moving frame to a good approximation, and we use this value for $U_{\text {roll }}$ to define the stream function $\Psi$, which is now a periodic function of time.

As the fluid flow in our model only depends on two spatial variables, the bifurcation to intrinsic time-dependence gives the system the third degree of freedom necessary to obtain chaotic fluid trajectories. These will initially appear around separatrices of the original steady flow, and will lead to exponential line stretching and possible fast dynamo processes (Bayly \& Childress 1988; Galloway \& Proctor 1992; Otani 1993). Figure $8(a, b)$ shows the flow with $R a=21 R a_{c} \simeq 7.8 \times 10^{5}$. As the flow is intrinsically time-dependent the pattern of $V$ and $\Psi$ will change periodically with time, as well as translating. The horizontal velocity produced by the convection now begins to dominate over the Ekman layer mean flow. The field at $q=75$ or $R m \simeq 5480$ is 

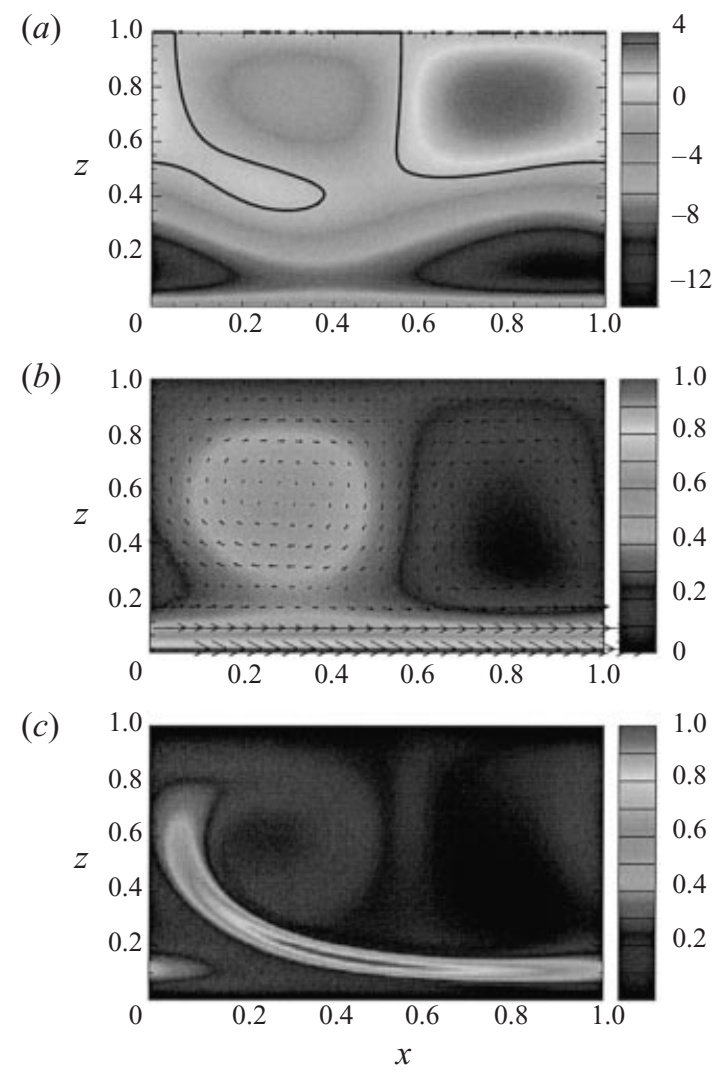

FIGURE 7. A flow resulting from a saturated convective instability near onset drives a dynamo with the parameter values $\vartheta=67.5^{\circ}, \tau=200, \epsilon=2.33^{\circ}, k_{c}=4.30, R e=30$ and $R a=4125 \simeq 1.1 R a_{c}$. For the magnetic field the Roberts number is taken as $q=50$ corresponding to $R m \simeq 500$, with the wavenumber $l=8.5$. It is shown as a time-snapshot in the $(\bar{x}, z)$-plane of $(a)$ the total velocity $V$ along the $\bar{y}$-axis, $(b)$ the total stream function $\Psi$, and $(c)$ the magnitude of the magnetic field.

shown in figure $8(c)$ and takes the form of sheets accumulating in the region close to the separatrices of the instantaneous form of $\Psi$ shown in figure $8(b)$. Those magnetic structures are similar to those obtained for fast dynamos in several idealized flows (Galloway \& Proctor 1992; Otani 1993; Ponty, Pouquet \& Sulem 1995; Kim et al. 1999).

For two different values of the Roberts number $q$, the magnetic growth rate is plotted against the magnetic wavenumber $l$ in figure 9 . The position of the dominant mode is largely independent of the Roberts number, as both curves have peaks situated around $l=8.5$. For this wavenumber, in figure 10 the magnetic growth rate $\sigma$ (left-hand side) or $\lambda$ (right-hand side) is plotted against the Roberts number $q$ (bottom scale) or, equivalently, the magnetic Reynolds number $R m$ (top scale). As $\mathrm{Rm}$ is increased, the dynamo growth rate increases to values of order the turnover time scale (see $\lambda$ ) and remains approximately constant, only dipping slightly for the highest values of $R m$. The values of $\lambda$ are also notably larger than those seen for the slow dynamos earlier in the paper (see figures 3 and 5). Although no numerical simulation can be completely definitive, this saturation of the growth rate $\lambda$ at values of order unity for high $R m$, the robustness of the maximum wavenumber $l$ in figure 9 , and the structure of the field in figure $8(c)$ all provide evidence indicating that this 
(a)

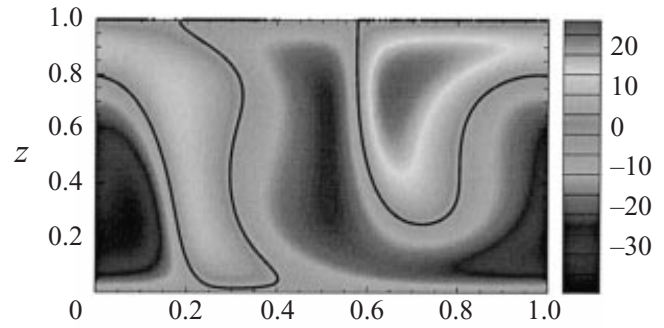

(b)

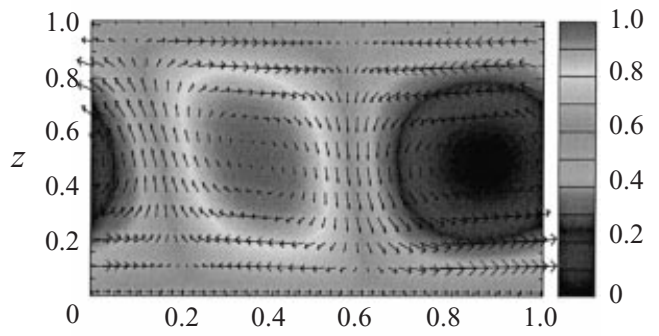

(c)

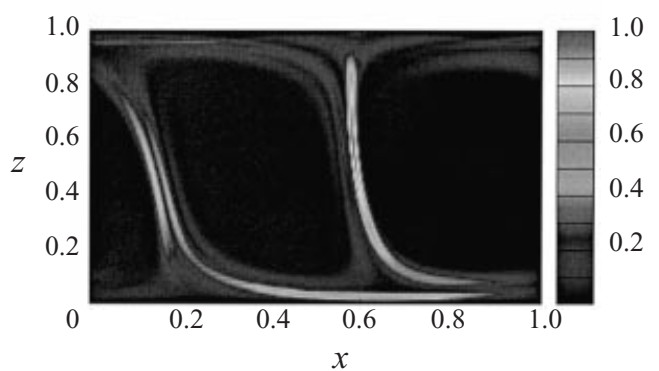

FIGURE 8. A flow resulting from a saturated convective instability far from onset drives a dynamo with the parameter values $\vartheta=67.5^{\circ}, \tau=200, \epsilon=2.33^{\circ}, k_{c}=4.30, R e=30$, and $R a=7.8 \times 10^{5} \simeq 21 R a_{c}$. The Roberts number is taken to be $q=75$ corresponding to $R m \simeq 5480$, with the wavenumber $l=8.5$. It is shown as a snapshot in the $(\bar{x}, z)$-plane of $(a)$ the total velocity $V$ along the $\bar{y}$-axis, $(b)$ the total stream function $\Psi$, and $(c)$ the magnitude of magnetic field.

flow is acting as a fast dynamo. That these diagnostics are a powerful indicator was argued recently by Kim et al. (1999).

\subsection{The effect of the shear flow}

Our system has two driving mechanisms, through heating and through motion of the bottom boundary. To understand something of the role of the latter mechanism, which imposes an overall shear flow on the system, we will describe a series of runs in which all parameters are kept constant, except for the Reynolds number. The parameters are as above, specifically $\vartheta=67.5^{\circ}, \tau=200, \epsilon=2.33^{\circ}, k_{c}=4.30$, $R a=7.8 \times 10^{5}, q=25$ and $l=8.5$. Figure $11(a, b)$ shows the magnetic growth rate $\lambda$ and the mean velocity $U$ (averaged over time and space), as $R e$ is varied from 0 to 200. The figure shows two distinct branches of solutions for the hydrodynamic system, marked with solid and dashed lines, with hysteresis around $R e=110$. The dynamo growth rate (figure 11a) varies markedly over the range of Re shown. In contrast, the mean velocity, $U$, (figure $11 b$ ) remains approximately constant, only increasing slightly with the Reynolds number. More useful is the amplitude of the intrinsic time-dependent part of the flow. A straightforward measure of this is to take the difference $\Delta U$ between minimum and maximum values of the velocity averaged 


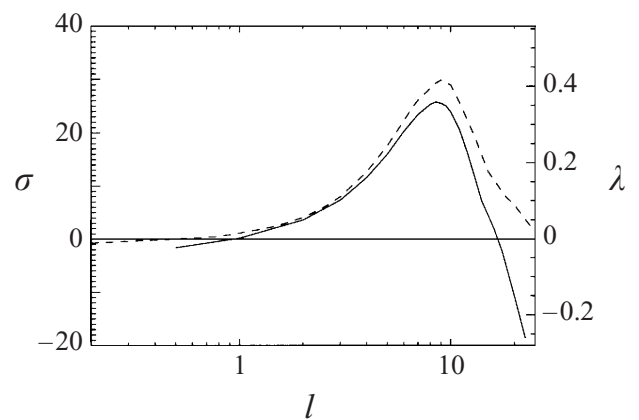

FIGURE 9. The magnetic field growth rate plotted against the wavenumber $l$ along the $\bar{y}$-axis, for two values of the Roberts number, $q=25$ (solid) and $q=50$ (dashed). Growth rates are shown in two different scalings: $\sigma$ on the left-hand side scaled with the thermal diffusion time, and $\lambda$ on the right-hand side with the turnover time scale. The flow is driven by the convective instability with the parameter values as in figure 8 .

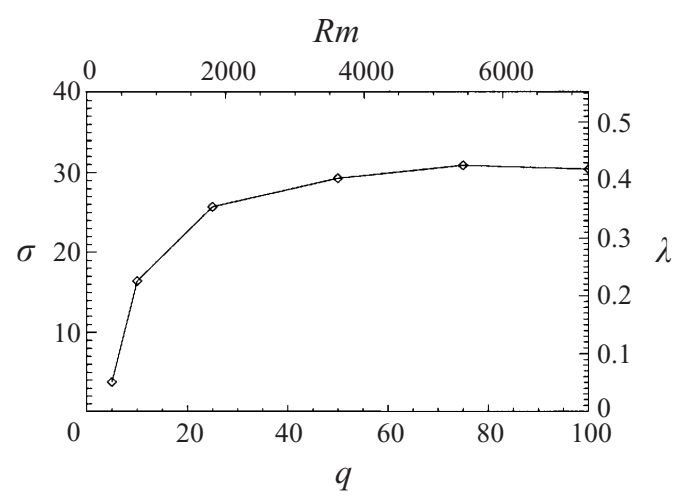

FigURE 10. The magnetic field growth rate $\sigma$ (left-hand scale) or $\lambda$ (right-hand scale), plotted against the Roberts number $q$ (lower scale) or $R m$ (upper scale). The flow is driven by convective instability with parameters as in figure 8 .

only over space, as it oscillates in time, and then to normalize this to form $\Delta U / U$, which is shown in figure $11(c)$.

On the first branch (solid line), there is moderate time-dependence at $R e=0$, which then becomes suppressed as the shear flow is increased (see figure 11c). Suppression of this time-dependence in a flow generally has the effect of reducing the size of regions of chaos in the system, and unsurprisingly causes the magnetic growth rate to decrease (Ponty et al. 1995). For Re between 70 and 109 the system loses intrinsic time-dependence, $\Delta U=0$, and is steady in an appropriate moving frame. In this region the growth rate increases a little, associated with a slow dynamo process in the steady flow. Around $R e=100$, the system undergoes a subcritical bifurcation (with some hysteresis as shown in figure 11), and the flow again becomes time-dependent. On this second branch (dashed line), the growth rate increases initially and then decreases, generally following the amplitude $\Delta U / U$ of the time-dependent part of the flow.

For three different values of the Reynolds number, $R e=0,50$ and 100 , the magnitude of the magnetic field is displayed in figure $12(a-c)$. Without shear, the magnetic field is present equally at the top and bottom of the layer (figure 12a). This 

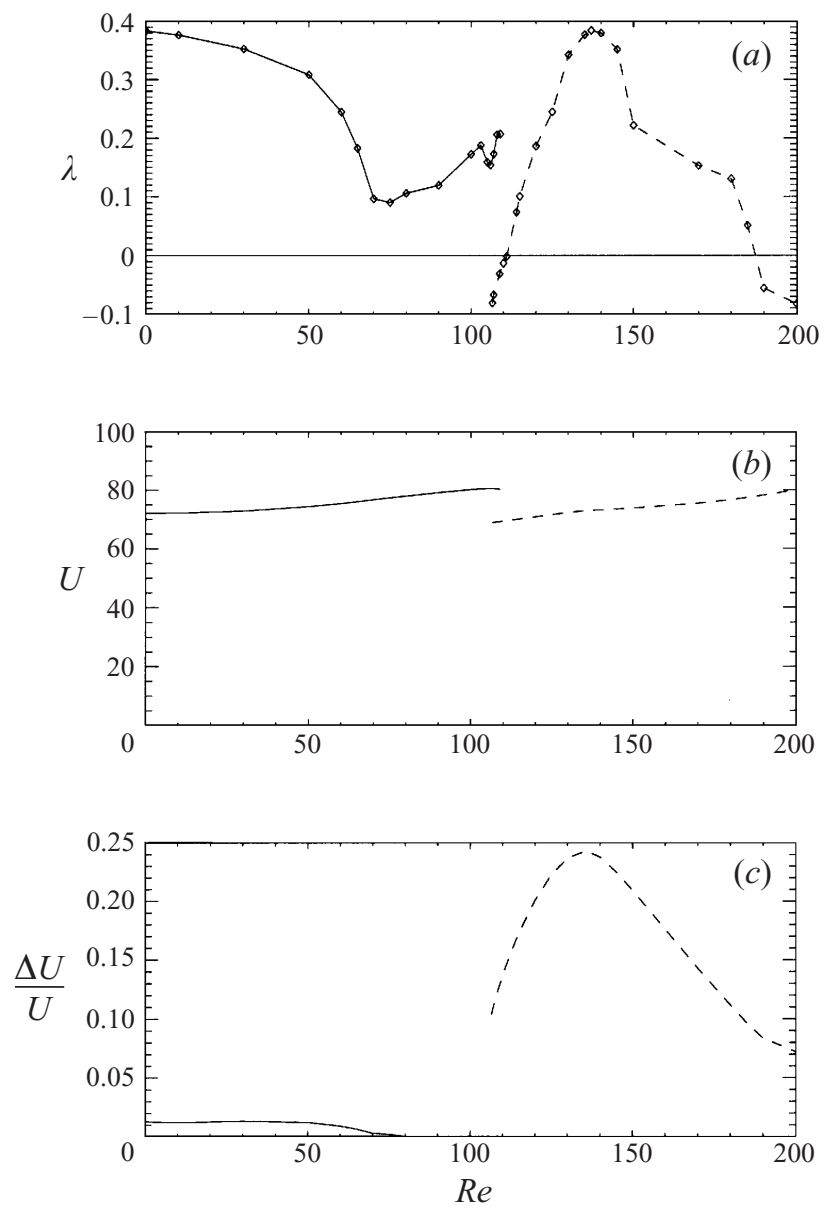

FIGURE 11. The effect of increasing shear. Plotted against the Reynolds number $R e$ are $(a)$ the magnetic field growth rate $\lambda$ (scaled with the turnover time), $(b)$ the time-averaged mean velocity $U$, and (c) the relative amplitude of the time dependent oscillations $\Delta U / U$. The solid and dashed line styles are associated with two distinct branches of the flow dynamics. The other parameter values are fixed, as given in the text.

represents dynamo action in a convecting rotating flow, with no other forcing, and is the configuration investigated by Matthews (1998) for moderate $R m$, but sufficiently large for dynamo action to occur. When the shear flow is introduced, the Ekman layer occurs only at the bottom and breaks the up-down symmetry. This is clear in figure $12(b)$ where the magnetic field appears to be preferentially located near the bottom of the layer. This localization increases as the shear is increased (figure 12c). Note that a bottom boundary condition of a perfect conductor (instead of an insulator) would tend to enhance this process (St Pierre 1993).

Although this picture is complicated in detail, the general behaviour is seen in many similar parameter regimes. The introduction of shear to a flow driven by convection tends overall to suppress the intrinsic time-dependence of the flow. This tends to reduce the chaotic stretching and so the fast dynamo growth rates; ultimately the time-dependence is turned off. As the shear is increased there is a clear tendency for the field to localize in the Ekman layer at the bottom boundary. 
(a)

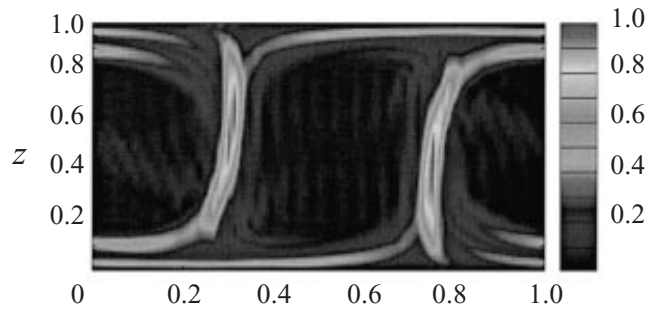

(b)

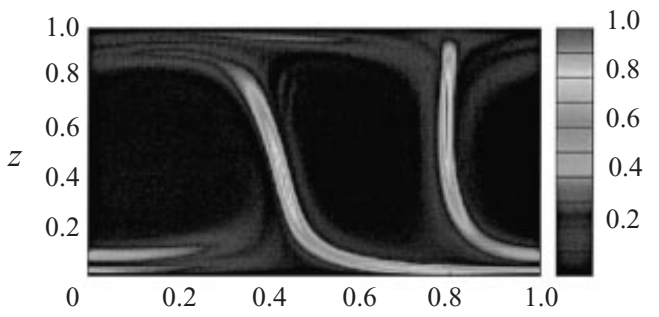

(c)

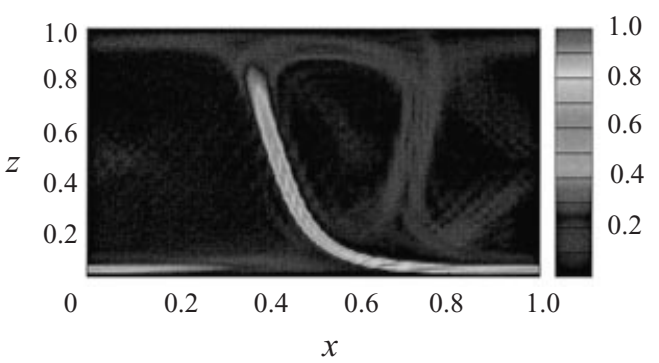

FiguRE 12. The magnitude of the magnetic field is shown for different values of the Reynolds number, (a) $R e=0,(b) R e=50$ and $(c) R e=100$. The common parameter values of the flow are $\vartheta=67.5^{\circ}, \tau=200, \epsilon=2.33^{\circ}, k_{c}=4.30, R a=7.8 \times 10^{5}, l=8.5$ and $q=25$.

\subsection{Vertical rotation $\vartheta=0^{\circ}$}

The second case we study shows the effect of a higher, vertical rotation rate with a thinner Ekman layer. Parameter values are $\vartheta=0^{\circ}, \tau=120, \epsilon=186.83^{\circ}, k_{c}=$ 4.79, $R e=120, R a=1.6 \times 10^{7} \simeq 26 R a_{c}$. The fluid system acquires intrinsic timedependence, implying the presence of chaotic fluid trajectories. Figure 13(a,b) shows a snapshot of the flow field, while figure 13(c) shows a growing magnetic field where the Roberts number is $q=20$ corresponding to $R m \simeq 2280$, with the wavenumber $l=8.0$. The magnetic field is again concentrated inside several magnetic sheets; one is located very close to the bottom boundary in the Ekman layer, the others lie in upand down-draughts. As in our first case of a convection-driven flow, we have evidence of fast dynamo amplification: the magnetic growth rate is displayed in figure 14, and levels off as $R m$ is increased.

\section{Discussion}

We have set up a model in which rotation, shear and convection drive complicated flows in a plane layer. The basic state, in which temperature and the shear flow depend just on the vertical coordinate, may be destabilized by purely hydrodynamic Ekman layer instabilities and convective instabilities, and these lead to a variety of nonlinearly equilibrated flows. In the case of the Ekman instability, the equilibrated 

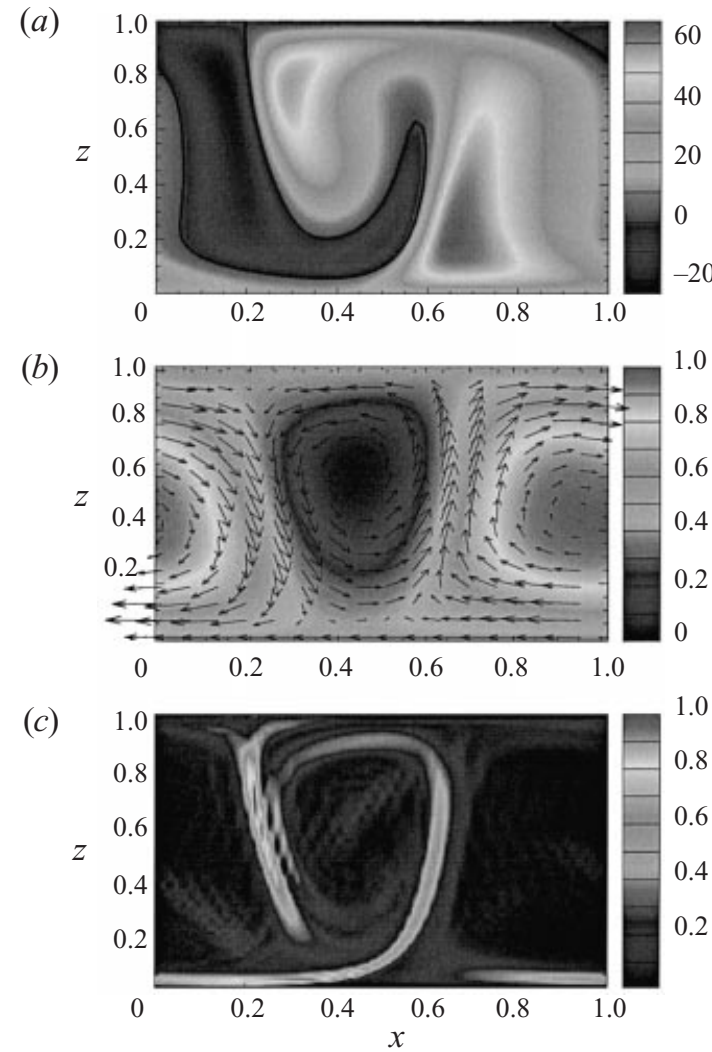

FiguRE 13. The flow is driven by the rotating convective instability far from the onset, with parameter values $\vartheta=0^{\circ}, \tau=120, \epsilon=186.83^{\circ}, k_{c}=4.79, R e=120, R a=1.6 \times 10^{7} \simeq 26 R a_{c}$. The Roberts number is taken $q=20$ corresponding to $R m \simeq 2280$ with the wavenumber $l=8.0$. It is shown as a snapshot in the $(\bar{x}, z)$-plane of $(a)$ the total velocity $V$ along the $\bar{y}$-axis, $(b)$ the total stream function $\Psi$, and $(c)$ the magnitude of magnetic field.

flows have the cat's eyes configuration and are steady in a co-moving frame. Dynamo action here may occur by the Ponomarenko (1973) dynamo mechanism or with fields associated with hyperbolic stagnation points and their connecting separatrices. The Ponomarenko mechanism is now well-understood: asymptotic high- $\mathrm{Rm}$ growth rates may be obtained in cases such as those seen in the simulations, when the stream surfaces are cylinders with arbitrary cross-section and the flow along the cylinders is arbitrary (Gilbert \& Ponty 2000).

Dynamo action associated with hyperbolic stagnation points and separatrices is rather more complicated. While dynamo action in flows with cat's eyes in the doubly periodic plane has been studied by Childress \& Soward (1989), there are nontrivial geometrical complications that arise in the flows of our plane layer model. In particular the layer is periodic in only one direction, along the cat's eyes, and the velocity perpendicular to the plane of the cat's eyes is not constant on stream lines. It remains an interesting asymptotic problem to obtain high- $R m$ growth rates in this more general situation.

In the case of flows driven by convection we obtain flows that are steady in a moving frame close to onset, and see similar mechanisms to those mentioned above. However, moving further from onset, the flows bifurcate and become periodic in 


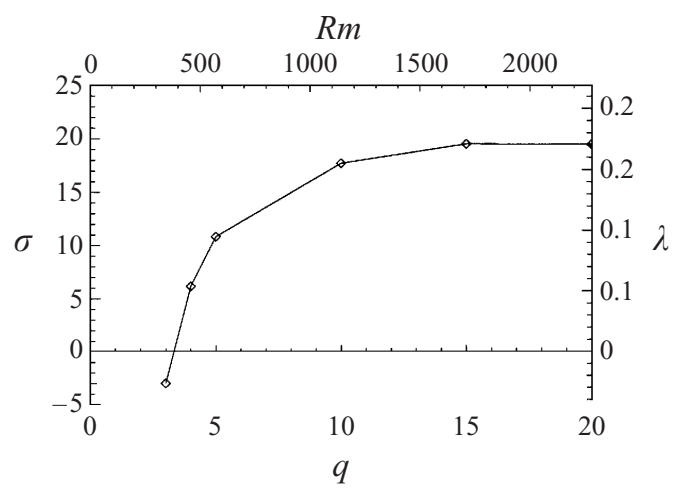

FIGURE 14. The magnetic field growth rate $\sigma$ (left-hand scale) or $\lambda$ (right-hand scale) plotted against the Roberts number $q$ (lower scale) or $R m$ (upper scale). The flow is driven by convective instability with parameters as in figure 13 .

a frame moving with a suitable velocity. In this case chaotic trajectories appear, initially close to separatrices. We have seen evidence for fast dynamo action in these flows, with maximum growth rates and dominant wavenumbers remaining relatively insensitive to magnetic Reynolds number in the limit of large $R m$, as far as our simulations allow.

Various dynamo scenarios occur depending on the relative importance of the different effects in our model. First we find that the combination of shear and vertical rotation in the basic state means that the flows generated after the first bifurcation of thermal or hydrodynamic instability, although two-dimensional, are sufficiently complex to act as dynamos; it is not necessary to go through further bifurcations to obtain dynamo action.

Secondly, we find that the shear in the Ekman layer tends to localize strong magnetic fields near the base of the plane layer. This is in agreement with Solar modelling in which a prescribed alpha effect and eddy diffusion are given (Deluca \& Gilman 1986, 1988; Parker 1993; Prautzsch 1993; Rüdiger \& Brandenburg 1995; Tobias 1995; Roald 1998), instead of resolving detailed fluid motions as we have done. Thirdly, note that in the case of the Ekman layer instability, at onset the fluid motions form cat's eyes within or close to the Ekman layer itself, and this is where the dynamo is located. On the other hand, when convective instability occurs, convective plumes go from the top to the bottom of the layer, and magnetic fields are more dispersed in the vertical (although strong at the bottom of the layer). This raises the difficult issue of whether in the Solar dynamo magnetic fields are generated by the interaction of convection and the tachocline, or through the dynamics of the tachocline itself. This question is unlikely to be answered until the tachocline is modelled in more detail (Spiegel \& Zahn 1992).

All the magnetic fields generated in our models have been of fairly small scale: our box is a local Cartesian approximation, and all the wavenumbers used have been of order unity or larger. In a future study it would be important to include the effects of the Lorentz force, which would allow nonlinear competition between different dynamo modes, and typically leads to the generation of magnetic fields on large scales. This would be important in extending our model towards the Solar context. We have also constrained the flows to be two-dimensional, and it would be interesting to extend our numerical code to three dimensions; however we would not be able to achieve the very large values of $R m$ studied in this paper. 
We are grateful to the Leverhulme Trust for the grant that supports Y.P. and this research. We enjoyed valuable discussions with Professor F. H. Busse, Professor C. A. Jones and Dr P. C. Matthews, and useful comments from the referees. The numerical calculations were performed using the computing facilities of the laboratory Cassini, Observatoire de Nice (France), provided by the program 'Simulations Interactives et Visualisation en Astronomie et Mécanique (SIVAM)' and the computing facilities of the parallel computer Ceres at the University of Exeter.

\section{REFERENCES}

BAYly, B. J. \& ChILDREss, S. 1988 Construction of fast dynamos using unsteady flows and maps in three dimensions. Geophys. Astrophys. Fluid Dyn. 44, 211-240.

Brandenburg, A., Nordlund, A., Pulkkinen, P., Stein, R. F. \& Tuominen, I. 1990 3D simulation of turbulent cyclonic magnetoconvection. Astron. Astrophys. 232, 277-291.

Brandenburg, A., Nordlund, A., Stein, R. F. \& Torkelsson, U. 1995 Dynamo generated turbulence and large scale magnetic fields in a Keplerian shear flow. Astrophys. J. 446, 741-754.

Brown, T. M., Christensen-Dalsgaard, J., Dziembowski, W. A., Goode, P. R., Gough, D. O. \& Morrow, C. 1989 Inferring the Sun's internal angular velocity from observed p-mode frequency splittings. Astrophys. J. 343, 526-546.

Busse, F. H. 1970 Thermal instability in rapidly rotating systems. J. Fluid Mech. 44, 441-460.

Busse, F. H. 1975 A model of the geodynamo. Geophys. J. R. Astr. Soc. 42, 437-459.

Busse, F. H. 1994 Convection driven zonal flows and vortices in the major planets. Chaos 4, 123-134.

Caldwell, D. R. \& Van AtTa, C. W. 1970 Characteristics of Ekman boundary layer instabilities. J. Fluid. Mech. 44, 79-95.

Cawthorne, A. B., Peffley, N. L. \& Lathrop, D. P. 2000 The approach to dynamo action in a liquid metal flow. Phys. Rev. Lett., submitted.

Childress, S. 1979 Alpha-effect in flux ropes and sheets. Phys. Earth Planet. Int. 20, 172-180.

Childress, S. \& Gilbert, A. D. 1995 Stretch, Twist, Fold: The Fast Dynamo. Springer.

Childress, S. \& Soward, A. M. 1972 Convection driven hydromagnetic dynamo. Phys. Rev. Lett. 29, 837-839.

Childress, S. \& Soward, A. M. 1989 Scalar transport and alpha-effect for a family of cat's-eyes flows. J. Fluid Mech. 205, 99-133.

Deluca, E. E. \& Gilman, P. A. 1986 Dynamo theory for the interface between the convection zone and the radiative interior of a star. Part I: Model equations and exact solutions. Geophys. Astrophys. Fluid Dyn. 37, 85-127.

Deluca, E. E. \& Gilman, P. A. 1988 Dynamo theory for the interface between the convection zone and the radiative interior of a star. Part II: Numerical solutions of the nonlinear equations. Geophys. Astrophys. Fluid Dyn. 43, 119-148.

Dziembowski, W., Goode, P. H. \& Libbrecht, K. G. 1989 The radial gradient in the Sun's rotation. Astrophys. J. 337, L53-L57.

FALLER, A. J. 1963 An experimental study of the instability of the laminar Ekman boundary layer. J. Fluid Mech. 15, 560-576.

Faller, A. J. \& KaYlor, R. E. 1966 A numerical study of the instability of laminar Ekman boundary layer flow. J. Atmos. Sci. 23, 466-480.

Galloway, D. J. \& Proctor, M. R. E. 1992 Numerical calculations of fast dynamo for smooth velocity field with realistic diffusion. Nature 356, 691-693.

Gailitis, A., Lielausis, O., Dement'ev, S., Platacis, E., Cifersons, A., Gerbeth, G., Gundrum, T., Stefani, F., Christen, M., Hänel, H. \& Gotthart, W. 2000 Dectection of a flow induced magnetic field eigenmode in the Riga dynamo facility. Phys. Rev. Lett., submitted.

Glatzmaier, G. A. 1984 Numerical simulations of stellar convective dynamos. I. The model and method. J. Comput. Phys. 55, 461-484.

Glatzmaier, G. A. $1985 a$ Numerical simulations of stellar convective dynamos. II. Field propagation in the convection zone. Astrophys. J. 291, 300-307.

Glatzmaier, G. A. $1985 b$ Numerical simulations of stellar convective dynamos. III. At the base of the convection zone. Geophys. Astrophys. Fluid Dyn. 31, 137-150. 
Gilbert, A. D. 1988 Fast dynamo action in the Ponomarenko dynamo Geophys. Astrophys. Fluid Dyn. 44, 241-258.

Gilbert, A. D. \& Ponty, Y. 2000 Slow Ponomarenko dynamos on stream surfaces. Geophys. Astrophys. Fluid Dyn., submitted.

Gilman, P. A. 1983 Dynamically consistent nonlinear dynamos driven by convection in a rotating spherical shell. II. Dynamos with cycles and strong feedbacks. Astrophys. J. Suppl. 53, 243-268.

Goode, P. R., Dziembowski, W. A., Korzennik, S. G. \& Rhodes, E. J. 1991 Astrophys. J. 367, 649-657.

Gottlieb, D. \& Orszag, S. A. 1977 Numerical Analysis of Spectral Methods: Theory and Applications. SIAM, Philadelphia.

Hoffmann, N. P. \& Busse, F. H. 1999 Instabilities of shear flows between two coaxial differentially rotating cones. Phys. Fluids 11, 1676-1678.

Hoffmann, N. P., Busse, F. H. \& Chen, W.-L. 1998 Transition to complex flows in the EkmanCouette layer. J. Fluid Mech. 366, 311-331.

Hurlburt, N. E., Toomre, J. \& Massaguer, J. M. 1986 Nonlinear compressible convection penetrating into stable layers and producing internal gravity waves. Astrophys. J. 311, 563-577.

Iooss, G., Nielsen, H. B. \& True, H. 1978 Bifurcation of the stationary Ekman flow into a stable periodic flow. Arch. Rat. Mech. Anal. 68, 227-256.

Jones, C. A. \& RoberTs, P. H. 2000 Convection driven dynamos in a rotating plane layer. J. Fluid Mech. 404, 311-343.

Kim, E.-J., Hughes, D. W. \& Soward, A. M. 1999 An investigation into high conductivity dynamo action driven by rotating convection. Geophys. Astrophys. Fluid Dyn. 91, 303-332.

KLAPPER, I. \& YounG, L. S. 1995 Bounds on the fast dynamo growth rate involving topological entropy. Commun. Math. Phys. 173, 623-646.

Krause, F. \& RÄDler, K.-H. 1980 Mean-Field Magnetohydrodynamics and Dynamo Theory. Pergamon.

Kropp, M. \& Busse, F. H. 1991 Thermal convection in differentially rotating systems. Geophys. Astrophys. Fluid Dyn. 61, 127-148.

Leibovich, S. \& LeLE, S. K. 1985 The influence of the horizontal component of Earth's angular velocity on the instability of the Ekman layer. J. Fluid Mech. 150, 41-87.

LiLly, D. K. 1966 On the instability of Ekman boundary flow. J. Atmos. Sci. 23, 481-494.

Matthews, P. C. 1998 Dynamo action in simple convective flows. Proc. R. Soc. Lond. A 455, 1829-1840.

Matthews, P. C. \& Cox, S. 1997 Linear stability of rotating convection in an imposed shear flow. J. Fluid Mech. 350, 271-293.

Melander, M. V. 1983 An algorithmic approach to the linear stability of the Ekman layer. J. Fluid Mech. 132, 283-293.

MoffatT, H. K. 1978 Magnetic Field Generation in Electrically Conducting Fluids. Cambridge University Press.

Nordlund, A. Brandenburg, A., Jennings, R. L., Rieutord, M., Ruokolainen, J., Stein, R. F. \& Tuominen, I. 1992 Dynamo action in stratified convection with overshoot. Astrophys. J. 392, 647-652.

Odier, P., Pinton, J. F. \& Fauve, S. 1998 Advection of the magnetic field by a turbulent swirling flow. Phys. Rev. E 58, 7397-7401.

Otani, N. F. 1993 A fast kinematic dynamo in two-dimensional time-dependent flows. J. Fluid Mech. 253, 327-340.

PARKer, E. N. 1979 Cosmical Magnetic Fields. Clarendon.

PARKER, E. N. 1993 A Solar dynamo surface wave at the interface between convection and nonuniform rotation. Astrophys. J. 408, 707-719.

Plunian, F., Marty, P. \& Alemany, A. 1999 Kinematic dynamo action in a network of screw motions; application to the core of a fast breeder reactor. J. Fluid Mech. 382, 137-154.

Ponomarenko, Y. B. 1973 On the theory of hydromagnetic dynamos Zh. Prikl. Mekh Tekh. Fiz. 6, $47-51$.

Ponty, Y., Gilbert, A. D. \& Soward, A. M. 2001 Rotating convective instability in an Ekman layer driven by a shear flow. In preparation.

Ponty, Y., Pouquet, A. \& Sulem, P. L. 1995 Dynamos in weakly chaotic two-dimensional flows. Geophys. Astrophys. Fluid Dyn. 79, 239-257. 
Prautzsch, T. 1993 The dynamo mechanism in the deep convection zone of the Sun. In Solar and Planetary Dynamos (ed. M. R. E. Proctor, P. C. Matthews \& A. M. Rucklidge), pp. 249-256. Cambridge University Press.

RoALD, C. B. 1998 A two-layer alpha-omega dynamo model with dynamic feedback on the omegaeffect. Mon. Not. R. Astron. Soc. 300, 397-410.

RoberTs, G. O. 1972 Dynamo action of fluid motions with two-dimensional periodicity. Phil. Trans. R. Soc. Lond. A 271, 411-454.

Roberts, P. H. 1992 The dynamo mechanism. In Production et Rôle du Champ Magnetique, pp. 61-117. Publ. Observatoire de Paris, Section de Meudon.

Rüdiger, G. \& BRAndenburg, A. 1995 A Solar dynamo in the overshoot layer - cycle period and butterfly diagram. Astron. Astrophys. 296, 557-566.

Ruzmaikin, A. A., Sokoloff, D. D. \& Shukurov, A. M. 1988 A hydromagnetic screw dynamo. J. Fluid Mech. 197, 39-56.

SowArD, A. M. 1987 Fast dynamo action in a steady flow. J. Fluid Mech. 180, 267-295.

Spiegel, E. A. \& Zahn, J.-P. 1992 The Solar tachocline. Astron. Astrophys. 265, 106-114.

St. Pierre, M. G. 1993 The strong field branch of the Childress-Soward Dynamo. In Solar and Planetary Dynamos (ed. M.R.E. Proctor, P.C. Matthews \& A.M. Rucklidge), pp. 295-302. Cambridge University Press.

Tobias, S. 1995 Nonlinear solar and stellar dynamos. PhD thesis, University of Cambridge.

Weiss, N. O. 1994 Solar and stellar dynamos. In Lectures on Solar and Planetary Dynamos (ed. M. R. E. Proctor \& A. D. Gilbert), pp. 59-95. Cambridge University Press.

Zeldovich, Ya. B., Ruzmaikin, A. A. \& Sokoloff, D. D. 1983 Magnetic Fields in Astrophysics. The Fluid Mechanics of Astrophysics and Geophysics, vol. 3. Gordon \& Breach Science Publishers. 